\title{
About Structure of Shannon Information Amount for Joint Filtering and Extrapolation Problem by Continuous-Discrete Memory Observations
}

\author{
Nikolas DYOMIN, Irina SAFRONOVA \\ Department of Applied Mathematics and Cybernetics, Tomsk State University \\ 36 Lenin ave., Tomsk, Russia \\ e-mail:svrhm@mail2000.ru \\ Svetlana ROZHKOVA \\ Department of Natural Sciences and Mathematics, Tomsk Polytechnic University \\ 30 Lenin ave., Tomsk, Russia \\ e-mail:svrhm@rambler.ru
}

Received: November 2003

\begin{abstract}
In this paper on basis of the results (Dyomin et al., 2003a) the structure of Shannon information amount in the joint filtering and extrapolation problem of the stochastic processes by continuous-discrete time memory observations is investigated. For particular class of processes with applying of the general results the problem of optimal transmission over the lag channels is considered and efficiency of filtering and extrapolation receptions under transmission over channels with memory or lag is investigated.
\end{abstract}

Key words: signal processing, filtering, extrapolation, information amount, memory.

\section{Introduction}

In (Dyomin et al., 2003a) with the use of the results (Abakumova et al., 1995a; 1995b; Dyomin et al., 1997; 2000) and the method of (Dyomin and Korotkevich, 1983; 1987; Liptser, 1974) on the basis of the stochastic analysis using Ito (Liptser and Shiryayev, 1977, 1978) and Ito-Ventzel (Ocone and Pardoux, 1989) formulae, the problem of defining joint information amount $I_{t, s}^{t}\left[x_{t}, \tilde{x}_{s}^{L} ; z_{0}^{t}, \eta_{0}^{m}\right]$ about the values $x_{t}$ of the nonobservable process in the current time $t$ and arbitrary number $\tilde{x}_{s}^{L}=\left[x_{s_{1}}, \cdots, x_{s_{L}}\right]$ of the future time points $\tilde{s}_{L}=\left[s_{1}, s_{2}, \cdots, s_{L}\right]$, which is in realization totality $\left\{z_{0}^{t} ; \eta_{0}^{m}\right\}$ of processes with continuous time $z_{t}$ and discrete time $\eta\left(t_{m}\right)$ with memory of arbitrary multiplicity $N$ relatively the nonobservable process, has been researched. This paper researches the structure of $I_{t, s}^{t}[\cdot]$ the point of view of representations $I_{t, s}^{t}\left[x_{t}, \tilde{x}_{s}^{L} ; z_{0}^{t}, \eta_{0}^{m}\right]$ through the information amounts $I_{t}\left[x_{t} ; z_{0}^{t}, \eta_{0}^{m}\right]$ and $I_{s}^{t}\left[\tilde{x}_{s}^{L} ; z_{0}^{t}, \eta_{0}^{m}\right]$ about the values $x_{t}$ and $\tilde{x}_{s}^{L}$ of the nonobservable process in the current $t$ and future $\tilde{s}_{L}$ time points contained in the realization totality $\left\{z_{0}^{t} ; \eta_{0}^{m}\right\}$ accordingly. The results of Theorems 3, 4 are presented in (Dyomin et al., 2003b) without proofs. 


\section{Statement of the Problem}

On the probability space $\left(\Omega, \mathcal{F}, F=\left(\mathcal{F}_{t}\right)_{t \geqslant 0}, \mathcal{P}\right)$ the nonobservable $n$-dimensional process $x_{t}$ (useful signal) and the observable $l$-dimensional process $z_{t}$ (an output signal of a continuous transmission channel) are defined by the stochastic differential equations (Liptser and Shiryayev, 1977, 1978)

$$
\begin{aligned}
& \mathrm{d} x_{t}=f\left(t, x_{t}\right) \mathrm{d} t+\Phi_{1}(t) \mathrm{d} w_{t}, \quad t \geqslant 0, \\
& \mathrm{~d} z_{t}=h\left(t, x_{t}, x_{\tau_{1}}, \cdots, x_{\tau_{N}}, z\right) \mathrm{d} t+\Phi_{2}(t, z) \mathrm{d} v_{t},
\end{aligned}
$$

and the observable $q$-dimensional process with discrete time $\eta\left(t_{m}\right)$ (an output signal of the discrete transmission channel) has the form

$$
\eta\left(t_{m}\right)=g\left(t_{m}, x_{t_{m}}, x_{\tau_{1}}, \cdots, x_{\tau_{N}}, z\right)+\Phi_{3}\left(t_{m}, z\right) \xi\left(t_{m}\right), \quad m=0,1, \ldots,
$$

where $0 \leqslant t_{0}<\tau_{N}<\cdots<\tau_{1}<t_{m} \leqslant t$.

REMARK 1. $1^{0}$ ) The assumptions and the notation of (Dyomin et al., 2003a) are used. $2^{0}$ ) With reference to the results (Dyomin et al., 2003a) the mark $\ll * \gg$, as a right upper index, will be given to formula, theorems, corollaries, propositions and remarks numbers.

The problem is stated: for a sequence of time moments $t<s_{1}<\ldots<s_{L}$ is to be found the relations defining a time evolution of joint information amount $I_{t, s}^{t}\left[x_{t}, \tilde{x}_{s}^{L} ; z_{0}^{t}, \eta_{0}^{m}\right]$ about the current $x_{t}$ and the future $\tilde{x}_{s}^{L}$ values of the nonobservable process which is contained in the realizations set $z_{0}^{t}=\left\{z_{\sigma} ; 0 \leqslant \sigma \leqslant t\right\}$ and $\eta_{0}^{m}=\left\{\eta\left(t_{0}\right), \eta\left(t_{1}\right), \ldots, \eta\left(t_{m}\right) ; t_{m} \leqslant t\right\}$ of the observable processes in the form of expansions representing $I_{t, s}^{t}[\cdot]$ through the information amounts $I_{t}\left[x_{t} ; z_{0}^{t}, \eta_{0}^{m}\right]$ and $I_{s}^{t}\left[\tilde{x}_{s}^{L} ; z_{0}^{t}, \eta_{0}^{m}\right]$ about the current and the future values of the nonobserved process, respectively.

Then, if suggested the existence of the probability densities

$$
\begin{aligned}
& p_{s}^{t}\left(x ; \tilde{x}^{L}\right)=\partial^{L+1} \mathcal{P}\left\{x_{t} \leqslant x ; \tilde{x}_{s}^{L} \leqslant \tilde{x}^{L} \mid z_{0}^{t}, \eta_{0}^{m}\right\} / \partial x \partial \tilde{x}^{L}, \\
& p\left(t, x ; \tilde{s}_{L}, \tilde{x}^{L}\right)=\partial^{L+1} \mathcal{P}\left\{x_{t} \leqslant x ; \tilde{x}_{s}^{L} \leqslant \tilde{x}^{L}\right\} / \partial x \partial \tilde{x}^{L}, \\
& p_{t}(x)=\partial \mathcal{P}\left\{x_{t} \leqslant x \mid z_{0}^{t}, \eta_{0}^{m}\right\} / \partial x, \quad p(t, x)=\partial \mathcal{P}\left\{x_{t} \leqslant x\right\} / \partial x, \\
& p_{s}^{t}\left(\tilde{x}^{L}\right)=\partial^{L} \mathcal{P}\left\{\tilde{x}_{s}^{L} \leqslant \tilde{x}^{L} \mid z_{0}^{t}, \eta_{0}^{m}\right\} / \partial \tilde{x}^{L}, p\left(\tilde{s}_{L}, \tilde{x}^{L}\right)=\partial^{L} \mathcal{P}\left\{\tilde{x}_{s}^{L} \leqslant \tilde{x}^{L}\right\} / \partial \tilde{x}^{L}, \\
& p_{s \mid t}^{t}\left(\tilde{x}^{L} \mid x\right)=\partial^{L} \mathcal{P}\left\{\tilde{x}_{s}^{L} \leqslant \tilde{x}^{L} \mid x_{t}=x, z_{0}^{t}, \eta_{0}^{m}\right\} / \partial \tilde{x}^{L}, \\
& p\left(\tilde{s}_{L}, \tilde{x}^{L} \mid t, x\right)=\partial^{L} \mathcal{P}\left\{\tilde{x}_{s}^{L} \leqslant \tilde{x}^{L} \mid x_{t}=x\right\} / \partial \tilde{x}^{L}, \\
& p_{t \mid s}^{t}\left(x \mid \tilde{x}^{L}\right)=\partial \mathcal{P}\left\{x_{t} \leqslant x \mid \tilde{x}_{s}^{L}=\tilde{x}^{L}, z_{0}^{t}, \eta_{0}^{m}\right\} / \partial x, \\
& p\left(t, x \mid \tilde{s}_{L}, \tilde{x}^{L}\right)=\partial \mathcal{P}\left\{x_{t} \leqslant x \mid \tilde{x}_{s}^{L}=\tilde{x}^{L}\right\} / \partial x
\end{aligned}
$$

the formulae take place

$$
I_{t, s}^{t}\left[x_{t}, \tilde{x}_{s}^{L} ; z_{0}^{t}, \eta_{0}^{m}\right]=I_{t}\left[x_{t} ; z_{0}^{t}, \eta_{0}^{m}\right]+I_{s \mid t}^{t}\left[\tilde{x}_{s}^{L} ; z_{0}^{t}, \eta_{0}^{m} \mid x_{t}\right],
$$




$$
\begin{aligned}
& I_{t, s}^{t}\left[x_{t}, \tilde{x}_{s}^{L} ; z_{0}^{t}, \eta_{0}^{m}\right]=I_{s}^{t}\left[\tilde{x}_{s}^{L} ; z_{0}^{t}, \eta_{0}^{m}\right]+I_{t \mid s}^{t}\left[x_{t} ; z_{0}^{t}, \eta_{0}^{m} \mid \tilde{x}_{s}^{L}\right], \\
& I_{t, s}^{t}\left[x_{t}, \tilde{x}_{s}^{L} ; z_{0}^{t}, \eta_{0}^{m}\right]=M\left\{\ln \left[p_{s}^{t}\left(x_{t} ; \tilde{x}_{s}^{L}\right) / p\left(t, x_{t} ; \tilde{s}_{L}, \tilde{x}_{s}^{L}\right)\right]\right\}, \\
& I_{t}\left[x_{t} ; z_{0}^{t}, \eta_{0}^{m}\right]=M\left\{\ln \left[p_{t}\left(x_{t}\right) / p\left(t, x_{t}\right)\right]\right\}, \\
& I_{s \mid t}^{t}\left[\tilde{x}_{s}^{L} ; z_{0}^{t}, \eta_{0}^{m} \mid x_{t}\right]=M\left\{\ln \left[p_{s \mid t}^{t}\left(\tilde{x}_{s}^{L} \mid x_{t}\right) / p\left(\tilde{s}_{L}, \tilde{x}_{s}^{L} \mid t, x_{t}\right)\right]\right\}, \\
& I_{s}^{t}\left[\tilde{x}_{s}^{L} ; z_{0}^{t}, \eta_{0}^{m}\right]=M\left\{\ln \left[p_{s}^{t}\left(\tilde{x}_{s}^{L}\right) / p\left(\tilde{s}_{L}, \tilde{x}_{s}^{L}\right)\right]\right\}, \\
& I_{t \mid s}^{t}\left[x_{t} ; z_{0}^{t}, \eta_{0}^{m} \mid \tilde{x}_{s}^{L}\right]=M\left\{\ln \left[p_{t \mid s}^{t}\left(x_{t} \mid \tilde{x}_{s}^{L}\right) / p\left(t, x_{t} \mid \tilde{s}_{L}, \tilde{x}_{s}^{L}\right)\right]\right\},
\end{aligned}
$$

where $I_{s \mid t}^{t}[\cdot]$ and $I_{t \mid s}^{t}[\cdot]$ are corresponding conditional information amounts (Gallager, 1968; Shannon and Weaver, 1949).

\section{Main Results}

REMARK 2. The information amounts $I_{t}\left[x_{t} ; z_{0}^{t}, \eta_{0}^{m}\right]$ and $I_{s}^{t}\left[\tilde{x}_{s}^{L} ; z_{0}^{t}, \eta_{0}^{m}\right]$ mentioned in expressions (2.12), (2.13) are defined by Corollary $1^{*}$ and Theorem $2^{*}$ (see $2^{0}$ of Remark 1).

Theorem 1. The information amount (2.14) can be represented in the form (2.12), where $I_{s \mid t}^{t}[\cdot]$ on the time intervals $t_{m} \leqslant t<t_{m+1}$ is determined by the equation

$$
\begin{aligned}
& \mathrm{d} I_{s \mid t}^{t}\left[\tilde{x}_{s}^{L} ; z_{0}^{t}, \eta_{0}^{m} \mid x_{t}\right] / \mathrm{d} t=(1 / 2) \operatorname{tr}\left[M \left\{R ^ { - 1 } ( t , z ) \left[\overline{h\left(\tilde{\tau}_{N}, z \mid x_{t}, \tilde{x}_{s}^{L}\right)}\right.\right.\right. \\
& \left.\left.\left.-\overline{h\left(\tilde{\tau}_{N}, z \mid x_{t}\right)}\right]\left[\overline{h\left(\tilde{\tau}_{N}, z \mid x_{t}, \tilde{x}_{s}^{L}\right)}-\overline{h\left(\tilde{\tau}_{N}, z \mid x_{t}\right)}\right]^{T}\right\}\right] \\
& -\frac{1}{2} \operatorname{tr}\left[Q ( t ) M \left\{\frac{\partial \ln p_{s \mid t}^{t}\left(\tilde{x}_{s}^{L} \mid x_{t}\right)}{\partial x_{t}}\left(\frac{\partial \ln p_{s \mid t}^{t}\left(\tilde{x}_{s}^{L} \mid x_{t}\right)}{\partial x_{t}}\right)^{T}\right.\right. \\
& \left.\left.-\frac{\partial \ln p\left(\tilde{s}_{L}, \tilde{x}_{s}^{L} \mid t, x_{t}\right)}{\partial x_{t}}\left(\frac{\partial \ln p\left(\tilde{s}_{L}, \tilde{x}_{s}^{L} \mid t, x_{t}\right)}{\partial x_{t}}\right)^{T}\right\}\right]
\end{aligned}
$$

with the initial condition

$$
\begin{aligned}
I_{s \mid t_{m}}^{t_{m}}\left[\tilde{x}_{s}^{L} ; z_{0}^{t_{m}}, \eta_{0}^{m} \mid x_{t_{m}}\right]= & I_{s \mid t_{m}}^{t_{m}-0}\left[\tilde{x}_{s}^{L} ; z_{0}^{t_{m}}, \eta_{0}^{m-1} \mid x_{t_{m}}\right] \\
& +\Delta I_{s \mid t_{m}}^{t_{m}}\left[\tilde{x}_{s}^{L} ; z_{0}^{t_{m}}, \eta\left(t_{m}\right) \mid x_{t_{m}}\right],
\end{aligned}
$$

where

$$
\begin{aligned}
& \overline{h\left(\tilde{\tau}_{N}, z \mid x, \tilde{x}^{L}\right)}=M\left\{h\left(t, x_{t}, \tilde{x}_{\tau}^{N}, z\right) \mid x_{t}=x, \tilde{x}_{s}^{L}=\tilde{x}^{L}, z_{0}^{t}, \eta_{0}^{m}\right\}, \\
& \overline{h\left(\tilde{\tau}_{N}, z \mid x\right)}=M\left\{h\left(t, x_{t}, \tilde{x}_{\tau}^{N}, z\right) \mid x_{t}=x, z_{0}^{t}, \eta_{0}^{m}\right\},
\end{aligned}
$$




$$
\begin{aligned}
& \Delta I_{s \mid t_{m}}^{t_{m}}\left[\tilde{x}_{s}^{L} ; z_{0}^{t_{m}}, \eta\left(t_{m}\right) \mid x_{t_{m}}\right]=M\left\{\ln \left[C\left(\eta\left(t_{m}\right), z \mid x_{t_{m}}, \tilde{x}_{s}^{L}\right) / C\left(\eta\left(t_{m}\right), z\right) \mid x_{t_{m}}\right]\right\}, \\
& C\left(\eta\left(t_{m}\right), z \mid x, \tilde{x}_{s}^{L}\right)=M\left\{C\left(x_{t_{m}}, \tilde{x}_{\tau}^{N}, \eta\left(t_{m}\right), z\right) \mid x_{t_{m}}=x, \tilde{x}_{s}^{L}=\tilde{x}^{L} ; z_{0}^{t_{m}}, \eta_{0}^{m-1}\right\}, \\
& C\left(\eta\left(t_{m}\right), z \mid x\right)=M\left\{C\left(x_{t_{m}}, \tilde{x}_{\tau}^{N}, \eta\left(t_{m}\right), z\right) \mid x_{t_{m}}=x ; z_{0}^{t_{m}}, \eta_{0}^{m-1}\right\}, \\
& C\left(x, \tilde{x}_{N}, \eta\left(t_{m}\right), z\right)=\exp \left\{-(1 / 2)\left[\eta\left(t_{m}\right)-g\left(t_{m}, x, \tilde{x}_{N}, z\right)\right]^{T} V^{-1}\left(t_{m}, z\right)\right. \\
& \left.\times\left[\eta\left(t_{m}\right)-g\left(t_{m}, x, \tilde{x}_{N}, z\right)\right]\right\},
\end{aligned}
$$

and $I_{s \mid t_{m}}^{t_{m}-0}[\cdot]=\lim I_{s \mid t}^{t}[\cdot]$ as $t \uparrow t_{m}$.

Proof. In accordance with (2.4), (2.6), and (2.8), we have

$$
p_{s \mid t}^{t}\left(\tilde{x}^{L} \mid x\right)=p_{s}^{t}\left(x ; \tilde{x}^{L}\right) / p_{t}(x) .
$$

Innovation process $\tilde{z}_{t}$, differential of which has the form $\mathrm{d} \tilde{z}_{t}=\mathrm{d} z_{t}-\overline{h(t, z)} \mathrm{d} t, \overline{h(t, z)}=$ $M\left\{h\left(t, x_{t}, \tilde{x}_{\tau}^{N}, z\right) \mid z_{0}^{t}, \eta_{0}^{m}\right\}$, is such that $\widetilde{Z}_{t}=\left(\tilde{z}_{t}, \mathcal{F}_{t}^{z}\right)$ is Wiener process with $M\left\{\tilde{z}_{t} \tilde{z}_{t}^{T} \mid \mathcal{F}_{t}^{z}\right\}=\int_{0}^{t} R(\tau, z) \mathrm{d} \tau$ (Liptser and Shiryayev, 1977; 1978). Therefore the differentiation of (3.9) according to Ito formula taking into account $(3.14)^{*},(3.28)^{*}$ (see $2^{0}$ of Remark 1) for $t_{m} \leqslant t<t_{m+1}$ gives

$$
\begin{aligned}
& \mathrm{d}_{t} p_{s \mid t}^{t}\left(\tilde{x}^{L} \mid x\right)=\left\{-L_{t, x}^{*}\left[p_{s \mid t}^{t}\left(\tilde{x}^{L} \mid x\right)\right]+p_{s \mid t}^{t}\left(\tilde{x}^{L} \mid x\right)\left[\overline{h\left(\tilde{\tau}_{N}, z \mid x, \tilde{x}^{L}\right)}\right.\right. \\
& \left.\left.-\overline{h\left(\tilde{\tau}_{N}, z \mid x\right)}\right]^{T} R^{-1}(t, z)\left[\overline{h(t, z)}-\overline{h\left(\tilde{\tau}_{N}, z \mid x\right)}\right]\right\} \mathrm{d} t \\
& \quad+p_{s \mid t}^{t}\left(\tilde{x}^{L} \mid x\right)\left[\overline{h\left(\tilde{\tau}_{N}, z \mid x, \tilde{x}^{L}\right)}-\overline{h\left(\tilde{\tau}_{N}, z \mid x\right)}\right]^{T} R^{-1}(t, z) \mathrm{d} \tilde{z}_{t} .
\end{aligned}
$$

The a priori density (2.9) is determined by the equation

$$
\mathrm{d}_{t} p\left(\tilde{s}_{L}, \tilde{x}^{L} \mid t, x\right)=-L_{t, x}^{*}\left[p\left(\tilde{s}_{L}, \tilde{x}^{L} \mid t, x\right)\right] \mathrm{d} t,
$$

which follows from (3.10). Differentiation according to Ito formula taking into account (3.10), (3.11) by analogy with $(3.18)^{*}$ gives

$$
\begin{aligned}
\mathrm{d}_{t} & \ln \left[\frac{p_{s \mid t}^{t}\left(\tilde{x}^{L} \mid x\right)}{p\left(\tilde{s}_{L}, \tilde{x}^{L} \mid t, x\right)}\right]=\left\{\left(\frac{L_{t, x}^{*}\left[p\left(\tilde{s}_{L}, \tilde{x}^{L} \mid t, x\right)\right]}{p\left(\tilde{s}_{L}, \tilde{x}^{L} \mid t, x\right)}-\frac{L_{t, x}^{*}\left[p_{s \mid t}^{t}\left(\tilde{x}^{L} \mid x\right)\right]}{p_{s \mid t}^{t}\left(\tilde{x}^{L} \mid x\right)}\right)\right. \\
& +\left[\overline{h\left(\tilde{\tau}_{N}, z \mid x, \tilde{x}^{L}\right)}-\overline{h\left(\tilde{\tau}_{N}, z \mid x\right)}\right]^{T} R^{-1}(t, z)\left[\left(\overline{h(t, z)}-\overline{h\left(\tilde{\tau}_{N}, z \mid x\right)}\right)\right. \\
& \left.\left.-\frac{1}{2}\left(\overline{h\left(\tilde{\tau}_{N}, z \mid x, \tilde{x}^{L}\right)}-\overline{h\left(\tilde{\tau}_{N}, z \mid x\right)}\right)\right]\right\} \mathrm{d} t \\
& +\left[\overline{h\left(\tilde{\tau}_{N}, z \mid x, \tilde{x}^{L}\right)}-\overline{h\left(\tilde{\tau}_{N}, z \mid x\right)}\right]^{T} R^{-1}(t, z) \mathrm{d} \tilde{z}_{t} .
\end{aligned}
$$


Applying to (3.12) Ito-Ventzel formula for $t_{m} \leqslant t<t_{m+1}$ by analogy with (3.19)*, we obtain

$$
\begin{aligned}
& \ln \left[\frac{p_{s \mid t}^{t}\left(\tilde{x}_{s}^{L} \mid x_{t}\right)}{p\left(\tilde{s}_{L}, \tilde{x}_{s}^{L} \mid t, x_{t}\right)}\right]=\left.\ln [\cdot]\right|_{t=t_{m}} \\
& +\frac{1}{2} \int_{t_{m}}^{t} \operatorname{tr}\left[R^{-1}(\sigma, z)\left[\overline{h\left(\tilde{\tau}_{N}, z \mid x_{\sigma}, \tilde{x}_{s}^{L}\right)}-\overline{h\left(\tilde{\tau}_{N}, z \mid x_{\sigma}\right)}\right][\cdot]^{T}\right] \mathrm{d} \sigma \\
& -\frac{1}{2} \int_{t_{m}}^{t} \operatorname{tr}\left[Q ( \sigma ) \left[\frac{\partial \ln p_{s \mid \sigma}^{\sigma}\left(\tilde{x}_{s}^{L} \mid x_{\sigma}\right)}{\partial x_{\sigma}}\left(\frac{\partial \ln p_{s \mid \sigma}^{\sigma}(\cdot)}{\partial x_{\sigma}}\right)^{T}\right.\right. \\
& \left.\left.\quad-\frac{\partial \ln p\left(\tilde{s}_{L}, \tilde{x}_{s}^{L} \mid \sigma, x_{\sigma}\right)}{\partial x_{\sigma}}\left(\frac{\partial \ln p(\cdot)}{\partial x_{\sigma}}\right)^{T}\right]\right] \mathrm{d} \sigma \\
& \quad \int_{t_{m}}^{t} \operatorname{tr}\left[R { } ^ { - 1 } ( \sigma , z ) [ \overline { h ( \tilde { \tau } _ { N } , z | x _ { \sigma } , \tilde { x } _ { s } ^ { L } ) } - \overline { h ( \tilde { \tau } _ { N } , z | x _ { \sigma } ) } ] \left[h\left(\sigma, x_{\sigma}, \tilde{x}_{\tau}^{N}, z\right)\right.\right. \\
& +\int_{t_{m}}^{t} \frac{\partial}{\partial x_{\sigma}} \ln \frac{p_{s \mid \sigma}^{\sigma}\left(\tilde{x}_{s}^{L} \mid x_{\sigma}\right)}{p\left(\tilde{s}_{L}, \tilde{x}_{s}^{L} \mid \sigma, x_{\sigma}\right)} \Phi_{1}(\sigma) \mathrm{d} \omega_{\sigma}+ \\
& +\int_{t_{m}}^{t}\left[\frac{\mathrm{h} \sigma\left(\tilde{\tau}_{N}, z \mid x_{\sigma}, \tilde{x}_{s}^{L}\right)}{h\left(\tilde{\tau}_{N}, z \mid x_{\sigma}, \tilde{x}_{s}^{L}\right)}-\overline{h\left(\tilde{\tau}_{N}, z \mid x_{\sigma}\right)}\right]^{T} R^{-1}(\sigma, z) \Phi_{2}(\sigma, z) d v_{\sigma} . \\
& \quad
\end{aligned}
$$

Since, in accordance with (3.3), (3.4), we have $M\left\{h\left(\sigma, x_{\sigma}, \tilde{x}_{\tau}^{N}, z\right)\right\}=$ $M\left\{M\left\{M\left\{M\left\{h(\cdot) \mid x_{\sigma}=x, \tilde{x}_{s}^{L}=\tilde{x}^{L}, z_{0}^{\sigma}, \eta_{0}^{m}\right\} \mid x_{\sigma}=x, z_{0}^{\sigma}, \eta_{0}^{m}\right\} \mid z_{0}^{\sigma}, \eta_{0}^{m}\right\}\right\}=$ $M\left\{M\left\{M\left\{\overline{h\left(\tilde{\tau}_{N}, z \mid x_{\sigma}, \tilde{x}_{s}^{L}\right)} \mid x_{\sigma}=x, z_{0}^{\sigma}, \eta_{0}^{m}\right\} \mid z_{0}^{\sigma}, \eta_{0}^{m}\right\}\right\}=M\left\{M\left\{\overline{h\left(\tilde{\tau}_{N}, z \mid x_{\sigma}\right)} \mid z_{0}^{\sigma}, \eta_{0}^{m}\right\}\right\}$, then

$$
\begin{aligned}
& M\left\{R^{-1}(\sigma, z)\left[\overline{h\left(\tilde{\tau}_{N}, z \mid x_{\sigma}, \tilde{x}_{s}^{L}\right)}-\overline{h\left(\tilde{\tau}_{N}, z \mid x_{\sigma}\right)}\right]\left[h\left(\sigma, x_{\sigma}, \tilde{x}_{\tau}^{N}, z\right)-\overline{h\left(\tilde{\tau}_{N}, z \mid x_{\sigma}, \tilde{x}_{s}^{L}\right)}\right]^{T}\right\} \\
& \quad=M\left\{R^{-1}(\sigma, z) M\left\{[\cdot][\cdot]^{T} \mid z_{0}^{\sigma}, \eta_{0}^{m}\right\}\right\}=O
\end{aligned}
$$

Taking the expectation of the left and the right part of (3.13) using (3.14) (see $1^{0}$ of Remark 1) and differentiating with respect to $t$ gives (3.1) by analogy with (3.8)* . Relations (3.9) and $(3.16)^{*},(3.29)^{*}$ imply that

$$
p_{s \mid t_{m}}^{t_{m}}\left(\tilde{x}^{L} \mid x\right)=\left[C\left(\eta\left(t_{m}\right), z \mid x, \tilde{x}^{L}\right) / C\left(\eta\left(t_{m}\right), z \mid x\right)\right] p_{s \mid t_{m}}^{t_{m}-0}\left(\tilde{x}^{L} \mid x\right)
$$


The substitution of (3.15) into (2.16) gives (3.2), (3.5). Expansion (2.12) for $I_{t, s}^{t}[\cdot]$ arise from using (3.9) in (2.14) taking into account $(2.15),(2.16)$ and $p\left(t, x ; \tilde{s}_{L}, \tilde{x}^{L}\right)=$ $p(t, x) p\left(\tilde{s}_{L}, \tilde{x}^{L} \mid t, x\right)$.

Theorem 2. The information amount (2.14) can be represented in the form (2.13), where $I_{t \mid s}^{t}[\cdot]$ on the time intervals $t_{m} \leqslant t<t_{m+1}$ is determined by the equation

$$
\begin{aligned}
\mathrm{d} I_{t \mid s}^{t}\left[x_{t} ; z_{0}^{t}, \eta_{0}^{m} \mid \tilde{x}_{s}^{L}\right] / \mathrm{d} t=(1 / 2) \operatorname{tr}\left[M \left\{R ^ { - 1 } ( t , z ) \left[\overline{h\left(\tilde{\tau}_{N}, z \mid x_{t}, \tilde{x}_{s}^{L}\right)}\right.\right.\right. \\
\left.\left.\left.-\overline{h\left(\tilde{\tau}_{N}, t, z \mid \tilde{x}_{s}^{L}\right)}\right]\left[\overline{h\left(\tilde{\tau}_{N}, z \mid x_{t}, \tilde{x}_{s}^{L}\right)}-\overline{h\left(\tilde{\tau}_{N}, t, z \mid \tilde{x}_{s}^{L}\right)}\right]^{T}\right\}\right] \\
-\frac{1}{2} \operatorname{tr}\left[Q ( t ) M \left\{\frac{\partial \ln p_{t \mid s}^{t}\left(x_{t} \mid \tilde{x}_{s}^{L}\right)}{\partial x_{t}}\left(\frac{\partial \ln p_{t \mid s}^{t}\left(x_{t} \mid \tilde{x}_{s}^{L}\right)}{\partial x_{t}}\right)^{T}\right.\right. \\
\left.\left.-\frac{\partial \ln p\left(t, x_{t} \mid \tilde{s}_{L}, \tilde{x}_{s}^{L}\right)}{\partial x_{t}}\left(\frac{\partial \ln p\left(t, x_{t} \mid \tilde{s}_{L}, \tilde{x}_{s}^{L}\right)}{\partial x_{t}}\right)^{T}\right\}\right] \\
+\operatorname{tr}\left[Q ( t ) M \left\{\left[\frac{\partial \ln p_{t \mid s}^{t}\left(x_{t} \mid \tilde{x}_{s}^{L}\right)}{\partial x_{t}}-\frac{\partial \ln p_{t}\left(x_{t}\right)}{\partial x_{t}}\right]\left(\frac{\partial \ln p_{t}\left(x_{t}\right)}{\partial x_{t}}\right)^{T}\right.\right. \\
\left.\left.-\left[\frac{\partial \ln p\left(t, x_{t} \mid \tilde{s}_{L}, \tilde{x}_{s}^{L}\right)}{\partial x_{t}}-\frac{\partial \ln p\left(t, x_{t}\right)}{\partial x_{t}}\right]\left(\frac{\partial \ln p\left(t, x_{t}\right)}{\partial x_{t}}\right)^{T}\right\}\right]
\end{aligned}
$$

with the initial condition

$$
\begin{aligned}
I_{t_{m} \mid s}^{t_{m}}\left[x_{t_{m}} ; z_{0}^{t_{m}}, \eta_{0}^{m} \mid \tilde{x}_{s}^{L}\right]= & I_{t_{m} \mid s}^{t_{m}-0}\left[x_{t_{m}} ; z_{0}^{t_{m}}, \eta_{0}^{m-1} \mid \tilde{x}_{s}^{L}\right] \\
& +\Delta I_{t_{m} \mid s}^{t_{m}}\left[x_{t_{m}} ; z_{0}^{t_{m}}, \eta\left(t_{m}\right) \mid \tilde{x}_{s}^{L}\right]
\end{aligned}
$$

where

$$
\begin{aligned}
& \overline{h\left(\tilde{\tau}_{N}, t, z \mid \tilde{x}^{L}\right)}=M\left\{h\left(t, x_{t}, \tilde{x}_{\tau}^{N}, z\right) \mid \tilde{x}_{s}^{L}=\tilde{x}^{L}, z_{0}^{t}, \eta_{0}^{m}\right\}, \\
& \Delta I_{t_{m} \mid s}^{t_{m}}\left[x_{t_{m}} ; z_{0}^{t_{m}}, \eta\left(t_{m}\right) \mid \tilde{x}_{s}^{L}\right] \\
& =M\left\{\ln \left[C\left(\eta\left(t_{m}\right), z \mid x_{t_{m}}, \tilde{x}_{s}^{L}\right) / C\left(\eta\left(t_{m}\right), z \mid \tilde{x}_{s}^{L}\right)\right]\right\}, \\
& C\left(\eta\left(t_{m}\right), z \mid \tilde{x}^{L}\right)=M\left\{C\left(x_{t_{m}}, \tilde{x}_{\tau}^{N}, \eta\left(t_{m}\right), z\right) \mid \tilde{x}^{L}=\tilde{x}^{L} ; z_{0}^{t_{m}}, \eta_{0}^{m-1}\right\}, \\
& \text { and } I_{t_{m} \mid s}^{t_{m}-0}[\cdot]=\lim I_{t \mid s}^{t}[\cdot] \text { as } t \uparrow t_{m} .
\end{aligned}
$$

Proof. From (2.4), (2.7) and (2.10), we have

$$
p_{t \mid s}^{t}\left(x \mid \tilde{x}^{L}\right)=p_{s}^{t}\left(x ; \tilde{x}^{L}\right) / p_{s}^{t}\left(\tilde{x}^{L}\right) .
$$


The differentiation of (3.21) according to Ito formula taking into account $(3.14)^{*},(3.37)^{*}$ and $(2.11)^{*}$ gives

$$
\begin{aligned}
& \mathrm{d}_{t} p_{t \mid s}^{t}\left(x \mid \tilde{x}^{L}\right)=\left\{\mathcal{L}_{t, x}\left[p_{t \mid s}^{t}\left(x \mid \tilde{x}^{L}\right) ; p_{t}(x)\right]\right. \\
& \quad+p_{t \mid s}^{t}\left(x \mid \tilde{x}^{L}\right)\left[\overline{h\left(\tilde{\tau}_{N}, z \mid x, \tilde{x}^{L}\right)}-\overline{h\left(\tilde{\tau}_{N}, t, z \mid \tilde{x}^{L}\right)}\right]^{T} R^{-1}(t, z) \\
& \left.\quad \times\left[\overline{h(t, z)}-\overline{h\left(\tilde{\tau}_{N}, t, z \mid \tilde{x}^{L}\right)}\right]\right\} \mathrm{d} t \\
& \quad+p_{t \mid s}^{t}\left(x \mid \tilde{x}^{L}\right)\left[\overline{h\left(\tilde{\tau}_{N}, z \mid x, \tilde{x}^{L}\right)}-\overline{h\left(\tilde{\tau}_{N}, t, z \mid \tilde{x}^{L}\right)}\right]^{T} R^{-1}(t, z) \mathrm{d} \tilde{z}_{t} .
\end{aligned}
$$

The a priori density (2.11) is determined by the equation

$$
\mathrm{d}_{t} p\left(t, x \mid \tilde{s}_{L}, \tilde{x}^{L}\right)=\mathcal{L}_{t, x}\left[p\left(t, x \mid \tilde{s}_{L}, \tilde{x}^{L}\right) ; p(t, x)\right] \mathrm{d} t,
$$

which follows from (3.22). It follows from (3.21), (3.16)*, (3.38)* that

$$
p_{t_{m} \mid s}^{t_{m}}\left(x \mid \tilde{x}^{L}\right)=\left[C\left(\eta\left(t_{m}\right), z \mid x, \tilde{x}^{L}\right) / C\left(\eta\left(t_{m}\right), z \mid \tilde{x}^{L}\right)\right] p_{t_{m} \mid s}^{t_{m}-0}\left(x \mid \tilde{x}^{L}\right) .
$$

The differentiation of (3.21) according to Ito formula taking into account (3.22), (3.23) and, then the use of Ito-Ventzel formula brings us to (3.16). We omit the transformations, since it is similar to transformations of (3.1) production. The substitution of (3.24) into (2.18) gives (3.17), (3.19). Expansion (2.13) for $I_{t, s}^{t}[\cdot]$ arise from using (3.21) in (2.14) taking into account (2.17), (2.18) and $p\left(t, x ; \tilde{s}_{L}, \tilde{x}^{L}\right)=p\left(\tilde{s}_{L}, \tilde{x}^{L}\right) p\left(t, x \mid \tilde{s}_{L}, \tilde{x}^{L}\right)$.

COROLLARY 1. The information amount (2.14) is determined by Theorem 1*

Proof. Since

$$
\begin{aligned}
& \ln p_{s \mid t}^{t}\left(\tilde{x}^{L} \mid x\right)=\ln p_{s}^{t}\left(x ; \tilde{x}^{L}\right)-\ln p_{t}(x), \\
& \ln p\left(\tilde{s}_{L}, \tilde{x}^{L} \mid t, x\right)=\ln p\left(t, x ; \tilde{s}_{L}, \tilde{x}^{L}\right)-\ln p(t, x), \\
& \ln p_{t \mid s}^{t}\left(x \mid \tilde{x}^{L}\right)=\ln p_{s}^{t}\left(x ; \tilde{x}^{L}\right)-\ln p_{s}^{t}\left(\tilde{x}^{L}\right), \\
& \ln p\left(t, x \mid \tilde{s}_{L}, \tilde{x}^{L}\right)=\ln p\left(t, x ; \tilde{s}_{L}, \tilde{x}^{L}\right)-\ln p\left(\tilde{s}_{L}, \tilde{x}^{L}\right),
\end{aligned}
$$

using (3.25) in (3.1) and (3.26) in (3.16) and, then substituting its equations and (3.23)*, $(3.32)^{*}$ into (2.12) and (2.13), respectively, we obtain $(3.8)^{*}$. The use of (3.2), (3.5), (3.15), (3.25), $(3.24)^{*},(3.25)^{*},(3.29)^{*}$ in $(2.12)$ or (3.17), (3.19), (3.24), (3.26), (3.33)*, $(3.34)^{*},(3.38)^{*}$ in $(2.13)$ gives $(3.9)^{*},(3.12)^{*}$.

REMARK 3. The research of $I_{t, s}^{t}[\cdot]$ structure can be realized in a different way. 
It is evident that

$$
\begin{aligned}
& \operatorname{tr}\left[M\left\{R^{-1}(t, z)\left[\overline{h\left(\tilde{\tau}_{N}, z \mid x_{t}, \tilde{x}_{s}^{L}\right)}-\overline{h(t, z)}\right][\cdot]^{T}\right\}\right] \operatorname{tr}\left[M\left\{R^{-1}(t, z)\left[\overline{h\left(\tilde{\tau}_{N}, z \mid x_{t}, \tilde{x}_{s}^{L}\right)}-\overline{h\left(\tilde{\tau}_{N}, z \mid x_{t}\right)}\right][\cdot]^{T}\right\}\right] \\
&+\operatorname{tr}\left[M\left\{R^{-1}(t, z)\left[\overline{h\left(\tilde{\tau}_{N}, z \mid x_{t}\right)}-\overline{h(t, z)}\right][\cdot]^{T}\right\}\right], \\
& M\left\{\ln \frac{C\left(\eta\left(t_{m}\right), z \mid x_{t_{m}}, \tilde{x}_{s}^{L}\right)}{C\left(\eta\left(t_{m}\right), z\right)}\right\}=M\left\{\ln \frac{C\left(\eta\left(t_{m}\right), z \mid x_{t_{m}}, \tilde{x}_{s}^{L}\right)}{C\left(\eta\left(t_{m}\right), z \mid x_{t_{m}}\right)}\right\} \\
& \quad+M\left\{\ln \frac{C\left(\eta\left(t_{m}\right), z \mid x_{t_{m}}\right)}{C\left(\eta\left(t_{m}\right), z\right)}\right\}, \\
& \operatorname{tr}\left[M\left\{R^{-1}(t, z)\left[\overline{h\left(\tilde{\tau}_{N}, z \mid x_{t}, \tilde{x}_{s}^{L}\right)}-\overline{h(t, z)}\right][\cdot]^{T}\right\}\right] \\
&=\operatorname{tr}\left[M\left\{R^{-1}(t, z)\left[\overline{h\left(\tilde{\tau}_{N}, z \mid x_{t}, \tilde{x}_{s}^{L}\right)}-\overline{h\left(\tilde{\tau}_{N}, t, z \mid \tilde{x}_{s}^{L}\right)}\right][\cdot]^{T}\right\}\right] \\
&+\operatorname{tr}\left[M\left\{R^{-1}(t, z)\left[\overline{h\left(\tilde{\tau}_{N}, t, z \mid \tilde{x}_{s}^{L}\right)}-\overline{h(t, z)}\right][\cdot]^{T}\right\}\right], \\
& M\left\{\ln \frac{C\left(\eta\left(t_{m}\right), z \mid x_{t_{m}}, \tilde{x}_{s}^{L}\right)}{C\left(\eta\left(t_{m}\right), z\right)}=M\left\{\ln \frac{C\left(\eta\left(t_{m}\right), z \mid x_{t_{m}}, \tilde{x}_{s}^{L}\right)}{C\left(\eta\left(t_{m}\right), z \mid \tilde{x}_{s}^{L}\right)}\right\}\right. \\
&+M\left\{\ln \frac{C\left(\eta\left(t_{m}\right), z \mid \tilde{x}_{s}^{L}\right)}{C\left(\eta\left(t_{m}\right), z\right)}\right\} .
\end{aligned}
$$

Then, the use of (3.27), (3.28), (3.25), or (3.29), (3.30), (3.26) in Theorem $1^{*}$ brings us to expansions for $I_{t, s}^{t}[\cdot]$ which along with (3.1), (3.2), $(3.23)^{*},(3.24)^{*}$ or (3.16), (3.17), $(3.32)^{*},(3.33)^{*}$ can be interpreted as expansions (2.12), (2.13).

\section{Conditionally-Gaussian Case}

We consider the case of conditions

$$
\begin{aligned}
& f(\cdot)=f(t)+F(t) x_{t}, \quad p_{0}(x)=\mathcal{N}\left\{x ; \mu_{0}, \Gamma_{0}\right\} \\
& h(\cdot)=h(t, z)+H_{0, N}(t, z) \tilde{x}_{t, \tau}^{N+1}, \quad g(\cdot)=g\left(t_{m}, z\right)+G_{0, N}\left(t_{m}, z\right) \tilde{x}_{t_{m}, \tau}^{N+1} \\
& H_{0, N}(\cdot)=\left[H_{0}(t, z) \vdots H_{1}(t, z) \vdots \ldots \vdots H_{N}(t, z)\right]=\left[H_{0}(t, z) \vdots H_{1, N}(t, z)\right] \\
& G_{0, N}(\cdot)=\left[G_{0}\left(t_{m}, z\right) \vdots G_{1}\left(t_{m}, z\right) \vdots \ldots \vdots G_{N}\left(t_{m}, z\right)\right]=\left[G_{0}\left(t_{m}, z\right) \vdots G_{1, N}\left(t_{m}, z\right)\right],
\end{aligned}
$$

when the property $(4.4)^{*}$, Proposition $2^{*}$, and Remark $3^{*}$ take place.

REMARK 4. The information amounts $I_{t}\left[x_{t} ; z_{0}^{t}, \eta_{0}^{m}\right]$ and $I_{s}^{t}\left[\tilde{x}_{s}^{L} ; z_{0}^{t}, \eta_{0}^{m}\right]$ mentioned in expressions (2.12), (2.13) are defined by Corollary $2^{*}$ and Theorem $4^{*}$. 
Theorem 3. The information amount (2.14) can be represented in the form (2.12) where $I_{s \mid t}^{t}[\cdot]$ on the time intervals $t_{m} \leqslant t<t_{m+1}$ is determined by the equation

$$
\begin{aligned}
\mathrm{d} I_{s \mid t}^{t} & {\left[\tilde{x}_{s}^{L} ; z_{0}^{t}, \eta_{0}^{m} \mid x_{t}\right] / \mathrm{d} t } \\
= & (1 / 2) \operatorname{tr}\left[M \left\{R ^ { - 1 } ( t , z ) \left[\widetilde{H}_{L+1}(t, z)\left(\widetilde{\Gamma}^{L+1}\left(t, \tilde{s}_{L}\right)\right)^{-1} \widetilde{H}_{L+1}^{T}(t, z)\right.\right.\right. \\
& \left.\left.\left.-\widetilde{H}_{0}(t, z) \Gamma^{-1}(t) \widetilde{H}_{0}^{T}(t, z)\right]\right\}\right] \\
& -(1 / 2) \operatorname{tr}\left[Q(t)\left[M\left\{\Gamma^{-1}\left(t \mid \tilde{s}_{L}\right)-\Gamma^{-1}(t)\right\}-\left[D^{-1}\left(t \mid \tilde{s}_{L}\right)-D^{-1}(t)\right]\right]\right]
\end{aligned}
$$

with the initial condition (3.2) where

$$
\begin{aligned}
& \Delta I_{s \mid t_{m}}^{t_{m}}[\cdot]=(1 / 2) M\left\{\ln \left[\left|\widetilde{\Gamma}^{L}\left(\tilde{s}_{L} \mid t_{m}-0\right)\right| /\left|\widetilde{\Gamma}^{L}\left(\tilde{s}_{L} \mid t_{m}\right)\right|\right]\right\}, \\
& \widetilde{\Gamma}^{L}\left(\tilde{s}_{L} \mid t\right)=\widetilde{\Gamma}^{L}\left(t, \tilde{s}_{L}\right)-\left(\widetilde{\Gamma}_{0, N+1}^{L}\left(t, \tilde{s}_{L}\right)\right)^{T} \Gamma^{-1}(t) \widetilde{\Gamma}_{0, N+1}^{L}\left(t, \tilde{s}_{L}\right),
\end{aligned}
$$

and $\widetilde{\Gamma}^{L+1}\left(t, \tilde{s}_{L}\right), \Gamma(t), \Gamma\left(t \mid \tilde{s}_{L}\right), \widetilde{H}_{0}(t, z), \widetilde{H}_{L+1}(t, z), D(t), D^{-1}\left(t \mid \tilde{s}_{L}\right)$ are determined by $(4.4)^{*}$ and Theorem $3^{*}$.

Proof. For $p_{\tau \mid t}^{t}\left(\tilde{x}_{N} \mid x\right)=\partial^{N} \mathcal{P}\left\{\tilde{x}_{\tau}^{N} \leqslant \tilde{x}_{N} \mid x_{t}=x, z_{0}^{t}, \eta_{0}^{m}\right\} / \partial \tilde{x}_{N}$ by analogy with $(4.13)^{*},(4.14)^{*}$, we have

$$
\begin{aligned}
& p_{\tau \mid t}^{t}\left(\tilde{x}_{N} \mid x\right)=\mathcal{N}\left\{\tilde{x} ; \tilde{\mu}_{N}\left(\tilde{\tau}_{N} \mid t\right), \widetilde{\Gamma}_{N}\left(\tilde{\tau}_{N} \mid t\right)\right\}, \\
& \tilde{\mu}_{N}\left(\tilde{\tau}_{N} \mid t\right)=\tilde{\mu}_{N}\left(\tilde{\tau}_{N}, t\right)+\widetilde{\Gamma}_{0 N}^{T}\left(\tilde{\tau}_{N}, t\right) \Gamma^{-1}(t)[x-\mu(t)], \\
& \widetilde{\Gamma}_{N}\left(\tilde{\tau}_{N} \mid t\right)=\widetilde{\Gamma}_{N}\left(\tilde{\tau}_{N}, t\right)-\widetilde{\Gamma}_{0 N}^{T}\left(\tilde{\tau}_{N}, t\right) \Gamma^{-1}(t) \widetilde{\Gamma}_{0 N}\left(\tilde{\tau}_{N}, t\right) .
\end{aligned}
$$

Formulae (3.3), (3.4), (4.1), (4.2), (4.6), and $(4.13)^{*},(4.14)^{*}$ imply that

$$
\begin{aligned}
& \overline{h\left(\tilde{\tau}_{N}, z \mid x\right)}=h(t, z)+H_{0}(t, z) x+H_{1, N}(t, z) \tilde{\mu}_{N}\left(\tilde{\tau}_{N} \mid t\right), \\
& \overline{h\left(\tilde{\tau}_{N}, z \mid x, \tilde{x}^{L}\right)}=h(t, z)+H_{0}(t, z) x+H_{1, N}(t, z) \tilde{\mu}_{N}\left(\tilde{\tau}_{N} \mid t, \tilde{s}_{L}\right) .
\end{aligned}
$$

Then, from (4.6), (4.7) and $(4.13)^{*},(4.14)^{*}$, we have

$$
\begin{aligned}
& \overline{h\left(\tilde{\tau}_{N}, z \mid x, \tilde{x}^{L}\right)}-\overline{h\left(\tilde{\tau}_{N}, z \mid x\right)}=H_{1, N}\left[\widetilde{\Gamma}_{N}^{L+1}\left(\widetilde{\Gamma}^{L+1}\right)^{-1}\left[\tilde{x}^{L+1}-\tilde{\mu}^{L+1}\left(t, \tilde{s}_{L}\right)\right]\right. \\
& \left.\quad-\widetilde{\Gamma}_{0 N}^{T} \Gamma^{-1}[x-\mu(t)]\right] .
\end{aligned}
$$

From (4.8) taking into account $(4.17)^{*}$, we obtain

$$
M\left\{\left[\overline{h\left(\tilde{\tau}_{N}, z \mid x_{t}, \tilde{x}_{s}^{L}\right)}-\overline{h\left(\tilde{\tau}_{N}, z \mid x_{t}\right)}\right][\cdot]^{T} \mid z_{0}^{t}, \eta_{0}^{m}\right\}
$$




$$
\begin{aligned}
= & H_{1, N} \widetilde{\Gamma}_{N}^{L+1}\left(\widetilde{\Gamma}^{L+1}\right)^{-1}\left(\widetilde{\Gamma}_{N}^{L+1}\right)^{T} H_{1, N}^{T}+H_{1, N} \widetilde{\Gamma}_{0 N}^{T} \Gamma^{-1} \widetilde{\Gamma}_{0 N} H_{1, N}^{T} \\
& -H_{1, N} \widetilde{\Gamma}_{N}^{L+1}\left(\widetilde{\Gamma}^{L+1}\right)^{-1}\left(\widetilde{\Gamma}_{0, N+1}^{L+1}\right)^{T} \Gamma^{-1} \widetilde{\Gamma}_{0 N} H_{1, N}^{T} \\
& -H_{1, N} \widetilde{\Gamma}_{0 N}^{T} \Gamma^{-1} \widetilde{\Gamma}_{0, N+1}^{L+1}\left(\widetilde{\Gamma}^{L+1}\right)^{-1}\left(\widetilde{\Gamma}_{N}^{L+1}\right)^{T} H_{1, N}^{T} .
\end{aligned}
$$

Formulae $(4.9)^{*}-(4.11)^{*},(4.14)^{*},(4.17)^{*},(4.19)^{*},(4.21)^{*}$ imply that

$$
\begin{aligned}
& H_{1, N} \widetilde{\Gamma}_{0 N}^{T}=\widetilde{H}_{0}-H_{0} \Gamma, \quad H_{1, N} \widetilde{\Gamma}_{N}^{L+1}=\widetilde{H}_{L+1}-H_{0} \widetilde{\Gamma}_{0, N+1}^{L+1}, \\
& \widetilde{H}_{L+1}\left(\widetilde{\Gamma}^{L+1}\right)^{-1}\left(\widetilde{\Gamma}_{0, N+1}^{L+1}\right)^{T}=\widetilde{H}_{0} .
\end{aligned}
$$

Substitution of (4.10) into (4.9) with the help of $(4.22)^{*}$ yields

$$
\begin{aligned}
& M\left\{\left[\overline{h\left(\tilde{\tau}_{N}, z \mid x_{t}, \tilde{x}_{s}^{L}\right)}-\overline{h\left(\tilde{\tau}_{N}, z \mid x_{t}\right)}\right][\cdot]^{T} \mid z_{0}^{t}, \eta_{0}^{m}\right\} \\
& \quad=\widetilde{H}_{L+1}(t, z)\left(\widetilde{\Gamma}^{L+1}\left(t, \tilde{s}_{L}\right)\right)^{-1} \widetilde{H}_{L+1}^{T}(t, z)-\widetilde{H}_{0}(t, z) \Gamma^{-1}(t) \widetilde{H}_{0}^{T}(t, z) .
\end{aligned}
$$

Relations (3.9), (3.21) imply that $\partial \ln p_{s \mid t}^{t}\left(\tilde{x}^{L} \mid x\right) / \partial x=\partial \ln p_{t \mid s}^{t}\left(x \mid \tilde{x}^{L}\right) / \partial x-$ $\partial \ln p_{t}(x) / \partial x$. According to $(4.25)^{*},(4.27)^{*}$, we have

$$
\partial \ln p_{s \mid t}^{t}\left(\tilde{x}^{L} \mid x\right) / \partial x=\Gamma^{-1}(t)[x-\mu(t)]-\Gamma^{-1}\left(t \mid \tilde{s}_{L}\right)\left[x-\mu\left(t \mid \tilde{s}_{L}\right)\right] .
$$

It follows from (4.12) and $(4.7)^{*},(4.24)^{*}$ that

$$
M\left\{\frac{\partial \ln p_{s \mid t}^{t}\left(\tilde{x}_{s}^{L} \mid x_{t}\right)}{\partial x_{t}}\left(\frac{\partial \ln p_{s \mid t}^{t}\left(\tilde{x}_{s}^{L} \mid x_{t}\right)}{\partial x_{t}}\right)^{T} \mid z_{0}^{t}, \eta_{0}^{m}\right\}=\Gamma^{-1}\left(t \mid \tilde{s}_{L}\right)-\Gamma^{-1}(t)
$$

Analogous calculations with regard to Remark $3^{*}$, give

$$
M\left\{\frac{\partial \ln p\left(\tilde{s}_{L}, \tilde{x}_{s}^{L} \mid t, x_{t}\right)}{\partial x_{t}}\left(\frac{\partial \ln p\left(\tilde{s}_{L}, \tilde{x}_{s}^{L} \mid t, x_{t}\right)}{\partial x_{t}}\right)^{T}\right\}=D^{-1}\left(t \mid \tilde{s}_{L}\right)-D^{-1}(t) .
$$

Substitution (4.11), (4.13), (4.14) in (3.1), taking into account the property $M\{\cdot\}=$ $M\left\{M\left\{\cdot \mid z_{0}^{t}, \eta_{0}^{m}\right\}\right\}$ gives (4.3). Since $p_{s \mid t}^{t}\left(\tilde{x}^{L} \mid x\right)=\mathcal{N}\left\{\tilde{x}^{L} ; \tilde{\mu}_{L}\left(\tilde{s}_{L} \mid t\right), \widetilde{\Gamma}^{L}\left(\tilde{s}_{L} \mid t\right)\right\}$, then according to (3.15) by analogy with $(4.32)^{*}$, we have

$$
M\left\{\ln \frac{C\left(\eta\left(t_{m}\right), z \mid x_{t_{m}}, \tilde{x}_{s}^{L}\right)}{C\left(\eta\left(t_{m}\right), z \mid x_{t_{m}}\right)}\right\}=\frac{1}{2} M\left\{\ln \frac{\left|\widetilde{\Gamma}^{L}\left(\tilde{s}_{L} \mid t_{m}-0\right)\right|}{\left|\widetilde{\Gamma}^{L}\left(\tilde{s}_{L} \mid t_{m}\right)\right|}\right\}
$$

Then, (4.4) arises from (3.5), (4.15) and (4.5) arises from (4.6)* taking into account the property for Gaussian density $p_{s}^{t}\left(x ; \tilde{x}^{L}\right)=p_{s}^{t}\left(\tilde{x}^{L+1}\right)=\mathcal{N}\left\{\tilde{x}^{L+1} ; \tilde{\mu}^{L+1}\left(t, \tilde{s}^{L}\right)\right.$, $\left.\widetilde{\Gamma}^{L+1}\left(t, \tilde{s}^{L}\right)\right\}$ (Liptser and Shiryayev, 1977; 1978). 
Theorem 4. The information amount (2.14) can be represented in the form (2.13) where $I_{t \mid s}^{t}[\cdot]$ on the time intervals $t_{m} \leqslant t<t_{m+1}$ is determined by the equation

$$
\begin{aligned}
& \mathrm{d} I_{t \mid s}^{t}\left[x_{t} ; z_{0}^{t}, \eta_{0}^{m} \mid \tilde{x}_{s}^{L}\right] / \mathrm{d} t=(1 / 2) \operatorname{tr}\left[M \left\{R^{-1}(t, z)\right.\right. \\
& \left.\left.\quad \times\left[\widetilde{H}_{L+1}(t, z)\left(\widetilde{\Gamma}^{L+1}\left(t, \tilde{s}_{L}\right)\right)^{-1} \widetilde{H}_{L+1}^{T}(t, z)-\widetilde{H}_{L}(t, z)\left(\widetilde{\Gamma}^{L}\left(t, \tilde{s}_{L}\right)\right)^{-1} \widetilde{H}_{L}^{T}(t, z)\right]\right\}\right] \\
& \quad-(1 / 2) \operatorname{tr}\left[Q(t)\left[M\left\{\Gamma^{-1}\left(t \mid \tilde{s}_{L}\right)\right\}-D^{-1}\left(t \mid \tilde{s}_{L}\right)\right]\right]
\end{aligned}
$$

with the initial condition (3.17) where

$$
\Delta I_{t_{m} \mid s}^{t_{m}}[\cdot]=(1 / 2) M\left\{\ln \left[\left|\Gamma\left(t_{m}-0 \mid \tilde{s}_{L}\right)\right| /\left|\Gamma\left(t_{m} \mid \tilde{s}_{L}\right)\right|\right]\right\},
$$

and $\widetilde{\Gamma}^{L}\left(t, \tilde{s}_{L}\right), \widetilde{\Gamma}^{L+1}\left(t, \tilde{s}_{L}\right), \Gamma\left(t \mid \tilde{s}_{L}\right), \widetilde{H}_{L}(t, z), \widetilde{H}_{L+1}(t, z), D\left(t \mid \tilde{s}_{L}\right)$ are determined by $(4.4)^{*}$ and Theorem $3^{*}$.

Proof. For $p_{t \mid s}^{t}\left(x \mid \tilde{x}^{L}\right)=\partial \mathcal{P}\left\{x_{t} \leqslant x \mid \tilde{x}_{s}^{L}=\tilde{x}^{L}, z_{0}^{t}, \eta_{0}^{m}\right\} / \partial x$ by analogy with (4.6)

$$
p_{t \mid s}^{t}\left(x \mid \tilde{x}^{L}\right)=\mathcal{N}\left\{x ; \mu\left(t \mid \tilde{s}_{L}\right), \Gamma\left(t \mid \tilde{s}_{L}\right)\right\},
$$

where $\mu\left(t \mid \tilde{s}_{L}\right)$ and $\Gamma\left(t \mid \tilde{s}_{L}\right)$ are determined by $(4.7)^{*},(4.24)^{*}$. Formulae (3.18), (4.1), (4.2), $(4.4)^{*}$, and $(4.37)^{*}$ imply that

$$
\begin{aligned}
& \overline{h\left(\tilde{\tau}_{N}, t, z \mid \tilde{x}^{L}\right)}=h(t, z)+H_{0}(t, z) \mu\left(t \mid \tilde{s}_{L}\right)+H_{1, N}(t, z) \tilde{\mu}_{N}\left(\tilde{\tau}_{N}, t \mid \tilde{s}_{L}\right), \\
& \tilde{\mu}_{N}\left(\tilde{\tau}_{N}, t \mid \tilde{s}_{L}\right)=\tilde{\mu}_{N}\left(\tilde{\tau}_{N}, t\right) \\
& \quad+\widetilde{\Gamma}_{N, N+1}^{L}\left(\tilde{\tau}_{N}, t, \tilde{s}_{L}\right)\left(\widetilde{\Gamma}^{L}\left(t, \tilde{s}_{L}\right)\right)^{-1}\left[\tilde{x}^{L}-\tilde{\mu}^{L}\left(t, \tilde{s}_{L}\right)\right] .
\end{aligned}
$$

Then, it follows from (4.7), (4.19) that

$$
\begin{aligned}
& \overline{h\left(\tilde{\tau}_{N}, z \mid x, \tilde{x}^{L}\right)}-\overline{h\left(\tilde{\tau}_{N}, t, z \mid \tilde{x}^{L}\right)}= \\
& \quad=H_{0}(t, z)\left[x-\mu\left(t \mid \tilde{s}_{L}\right)\right]+H_{1, N}(t, z)\left[\tilde{\mu}_{N}\left(\tilde{\tau}_{N} \mid t, \tilde{s}_{L}\right)-\tilde{\mu}_{N}\left(\tilde{\tau}_{N}, t \mid \tilde{s}_{L}\right)\right] .
\end{aligned}
$$

Analogously to (4.9) the use of $(4.13)^{*},(4.24)^{*},(4.20)$ in (4.21) gives

$$
\begin{aligned}
M\{ & {\left.\left[\overline{h\left(\tilde{\tau}_{N}, z \mid x_{t}, \tilde{x}_{s}^{L}\right)}-\overline{h\left(\tilde{\tau}_{N}, t, z \mid \tilde{x}_{s}^{L}\right)}\right][\cdot]^{T} \mid z_{0}^{t}, \eta_{0}^{m}\right\} } \\
= & H_{0}\left[\Gamma-\widetilde{\Gamma}_{0, N+1}^{L+1}\left(\widetilde{\Gamma}^{L+1}\right)^{-1}\left(\widetilde{\Gamma}_{0, N+1}^{L+1}\right)^{T}\right] H_{0}^{T} \\
& -H_{0} \widetilde{\Gamma}_{0, N+1}^{L}\left(\widetilde{\Gamma}^{L}\right)^{-1} \widetilde{H}_{L}^{T}+\widetilde{H}_{L+1}\left(\widetilde{\Gamma}^{L+1}\right)^{-1} \widetilde{H}_{L+1}^{T} \\
& -\widetilde{H}_{L+1}\left(\widetilde{\Gamma}^{L+1}\right)^{-1} \widetilde{\Gamma}_{N+1}^{L+1}\left(\widetilde{\Gamma}^{L}\right)^{-1} \widetilde{H}_{L}^{T}+H_{0} \widetilde{\Gamma}_{0, N+1}^{L+1}\left(\widetilde{\Gamma}^{L+1}\right)^{-1} \widetilde{\Gamma}_{N+1}^{L+1}\left(\widetilde{\Gamma}^{L}\right)^{-1} \widetilde{H}_{L}^{T} \\
& -\widetilde{H}_{L}\left(\widetilde{\Gamma}^{L}\right)^{-1}\left(\widetilde{\Gamma}_{0, N+1}^{L}\right)^{T} H_{0}^{T}-\widetilde{H}_{L}\left(\widetilde{\Gamma}^{L}\right)^{-1}\left(\widetilde{\Gamma}_{N+1}^{L+1}\right)^{T}\left(\widetilde{\Gamma}^{L+1}\right)^{-1} \widetilde{H}_{L+1}^{T}
\end{aligned}
$$




$$
\begin{aligned}
& +\widetilde{H}_{L}\left(\widetilde{\Gamma}^{L}\right)^{-1}\left(\widetilde{\Gamma}_{N+1}^{L+1}\right)^{T}\left(\widetilde{\Gamma}^{L+1}\right)^{-1}\left(\widetilde{\Gamma}_{0, N+1}^{L+1}\right)^{T} H_{0}^{T}+\widetilde{H}_{L}\left(\widetilde{\Gamma}^{L}\right)^{-1} \widetilde{H}_{L}^{T}, \\
\widetilde{\Gamma}_{N+1}^{L+1}\left(t, \tilde{s}_{L}\right)=M\left\{\left[\tilde{x}_{s}^{L+1}-\tilde{\mu}^{L+1}\left(t, \tilde{s}_{L}\right)\right]\left[\tilde{x}_{s}^{L}-\tilde{\mu}^{L}\left(t, \tilde{s}_{L}\right)\right]^{T} \mid z_{0}^{t}, \eta_{0}^{m}\right\} & \\
= & {\left[\begin{array}{c}
\widetilde{\Gamma}_{0, N+1}^{L}\left(t, \tilde{s}_{L}\right) \\
\widetilde{\Gamma}^{L}\left(t, \tilde{s}_{L}\right)
\end{array}\right] . }
\end{aligned}
$$

By analogy with $(4.22)^{*}$ using $(4.9)^{*},(4.17)^{*},(4.19)^{*},(4.21)^{*}$ and $(4.23)$, we obtain

$$
\begin{aligned}
& \widetilde{\Gamma}_{0, N+1}^{L+1}\left(t, \tilde{s}_{L}\right)\left(\widetilde{\Gamma}^{L+1}\left(t, \tilde{s}_{L}\right)\right)^{-1} \widetilde{\Gamma}_{N+1}^{L+1}\left(t, \tilde{s}_{L}\right)=\widetilde{\Gamma}_{0, N+1}^{L}\left(t, \tilde{s}_{L}\right), \\
& \widetilde{H}_{L+1}(t, z)\left(\widetilde{\Gamma}^{L+1}\left(t, \tilde{s}_{L}\right)\right)^{-1} \widetilde{\Gamma}_{N+1}^{L+1}\left(t, \tilde{s}_{L}\right)=\widetilde{H}_{L}(t, z) .
\end{aligned}
$$

Substitution of (4.24), (4.25) and (4.22)* into (4.22) gives

$$
\begin{aligned}
& M\left\{\left[\overline{h\left(\tilde{\tau}_{N}, z \mid x_{t}, \tilde{x}_{s}^{L}\right)}-\overline{h\left(\tilde{\tau}_{N}, t, z \mid \tilde{x}_{s}^{L}\right)}\right][\cdot]^{T} \mid z_{0}^{t}, \eta_{0}^{m}\right\} \\
& =\widetilde{H}_{L+1}(t, z)\left(\widetilde{\Gamma}^{L+1}\left(t, \tilde{s}_{L}\right)\right)^{-1} \widetilde{H}_{L+1}^{T}(t, z) \\
& \quad-\widetilde{H}_{L}(t, z)\left(\widetilde{\Gamma}^{L}\left(t, \tilde{s}_{L}\right)\right)^{-1} \widetilde{H}_{L}^{T}(t, z) .
\end{aligned}
$$

The use of $(3.26),(4.26)^{*},(4.30)^{*},(4.31)^{*}$ yields that

$$
\begin{aligned}
& M\left\{\left[\partial \ln p_{t \mid s}^{t}\left(x_{t} \mid \tilde{x}_{s}^{L}\right) / \partial x_{t}\right]\left[\partial \ln p_{t \mid s}^{t}\left(x_{t} \mid \tilde{x}_{s}^{L}\right) / \partial x_{t}\right]^{T} \mid z_{0}^{t}, \eta_{0}^{m}\right\}=\Gamma^{-1}\left(t \mid \tilde{s}_{L}\right), \\
& M\left\{\left[\partial \ln p\left(t, x_{t} \mid \tilde{s}_{L}, \tilde{x}_{s}^{L}\right) / \partial x_{t}\right]\left[\partial \ln p\left(t, x_{t} \mid \tilde{s}_{L}, \tilde{x}_{s}^{L}\right) / \partial x_{t}\right]^{T}\right\}=D^{-1}\left(t \mid \tilde{s}_{L}\right), \\
& M\left\{\left[\partial \ln p_{t \mid s}^{t}\left(x_{t} \mid \tilde{x}_{s}^{L}\right) / \partial x_{t}-\partial \ln p_{t}\left(x_{t}\right) / \partial x_{t}\right]\left[\partial \ln p_{t}\left(x_{t}\right) / \partial x_{t}\right]^{T} \mid z_{0}^{t}, \eta_{0}^{m}\right\}=O, \\
& M\left\{\left[\partial \ln p\left(t, x_{t} \mid \tilde{s}_{L}, \tilde{x}_{s}^{L}\right) / \partial x_{t}-\partial \ln p\left(t, x_{t}\right) / \partial x_{t}\right]\left[\partial \ln p\left(t, x_{t}\right) / \partial x_{t}\right]^{T}\right\}=O .(4.27)
\end{aligned}
$$

Substitution of (4.26), (4.27) into (3.16), taking into account $M\{\cdot\}=M\left\{M\left\{\cdot \mid z_{0}^{t}, \eta_{0}^{m}\right\}\right\}$ gives (4.16). The use of (4.18) in (3.19), taking into account (3.24) analogously to (4.4) brings us to (4.17).

COROLlaRY 2. The information amount (2.14) is determined by the Theorem $3^{*}$.

Proof. Substitution of (4.3), (4.33)* into (2.12) or substitution of (4.16), (4.35)* into (2.13) brings us to $(4.5)^{*}$. According to $(4.5)$ and $(4.6)^{*},(4.7)^{*}$, we have (Gantmakher, 1988)

$$
\left|\widetilde{\Gamma}^{L+1}\left(t, \tilde{s}_{L}\right)\right|=|\Gamma(t)| \cdot\left|\widetilde{\Gamma}^{L}\left(\tilde{s}_{L} \mid t\right)\right|,\left|\widetilde{\Gamma}^{L+1}\left(t, \tilde{s}_{L}\right)\right|=\left|\widetilde{\Gamma}^{L}\left(t, \tilde{s}_{L}\right)\right| \cdot\left|\Gamma\left(t \mid \tilde{s}_{L}\right)\right| .
$$

The use of (4.28) in (3.2), (4.4) or (3.17), (4.17) taking into account (2.12) or (2.13) brings us to $(4.12)^{*}$. 


\section{Optimal Transmission of the Gaussian Markov Process over the Lag Channels by the Silent Feedback}

In (Dyomin et al., 2003a) the problem of transmission of a stochastic signal over the continuous-discrete channel with the memory of a single multiplicity $\left(N=1, \tau_{1}=\tau\right)$ has been considered. This item is devoted to the problem of optimal transmission when in continuous and discrete channels the past signal value $x_{\tau}$ is transmitted, i.e., continuous and discrete channels are the transmission channels with lag. Case of a continuous channel with memory and a discrete one with lag and case of a discrete channel with memory and a continuous one with lag are considered. A comparison of efficiency of discrete channels with memory and lag is made.

The signal $x_{t}$, an output message of the continuous transmission channel $z_{t}$ and an output message of the discrete transmission channel $\eta\left(t_{m}\right)$ are scalar and defined in accordance with (2.1)-(2.3), (4.1), (4.2) in the form

$$
\begin{aligned}
& \mathrm{d} x_{t}=F(t) x_{t} \mathrm{~d} t+\Phi_{1}(t) \mathrm{d} w_{t}, \quad p_{0}(x)=\mathcal{N}\left\{x ; \mu_{0}, \gamma_{0}\right\}, \\
& \mathrm{d} z_{t}=h\left(t, x_{\tau}, z\right) \mathrm{d} t+\Phi_{2}(t) \mathrm{d} v_{t}, \quad \eta\left(t_{m}\right)=g\left(t_{m}, x_{\tau}, z\right)+\Phi_{3}\left(t_{m}\right) \xi\left(t_{m}\right),
\end{aligned}
$$

$0 \leqslant t_{0}<\tau<t_{m} \leqslant t$

Problem formulation: in the class of coding functionals $\mathcal{K}^{1}=\left\{\mathcal{H}^{1} ; \mathcal{G}^{1}\right\}=$ $\{h(\cdot) ; g(\cdot)\}$, satisfying energy limitations

$$
M\left\{h^{2}\left(t, x_{\tau}, z\right)\right\} \leqslant \tilde{h}(t), \quad M\left\{g^{2}\left(t_{m}, x_{\tau}, z\right)\right\} \leqslant \tilde{g}\left(t_{m}\right),
$$

the functionals $h^{0}(\cdot)$ and $g^{0}(\cdot)$, which provide the minimal decoding error $\Delta^{0}(t)=$ $\inf \Delta(t)$ with regard to a filtering problem, are to be found, where $\Delta(t)=M\left\{\left[x_{t}-\right.\right.$ $\left.\hat{x}(t, z, \eta)]^{2}\right\}$ is the mean-root-square error of filtering estimate $\hat{x}(t, z, \eta)$ of the process $x_{t}$ corresponding message $\left\{z_{0}^{t} ; \eta_{0}^{m}\right\}$ accepted by the given $h(\cdot)$ and $g(\cdot)$.

Since given $h(\cdot)$ and $g(\cdot)$, a posteriori mean $\mu(t)=M\left\{x_{t} \mid z_{0}^{t}, \eta_{0}^{m}\right\}$ (Liptser and Shiryayev, 1977; 1978) is optimal in mean-root-square sense filtering estimate, then $\Delta(t) \geqslant M\{\gamma(t)\}$, where $\gamma(t)=M\left\{\left[x_{t}-\mu(t)\right]^{2} \mid z_{0}^{t}, \eta_{0}^{m}\right\}$. Thus, we have $\Delta^{0}(t)=$ $\inf M\{\gamma(t)\}$.

REMARK 5. It is obvious that, up to the moment $\tau$ when $0 \leqslant t_{0} \leqslant t_{m} \leqslant t<\tau$, we have $h(\cdot)=h\left(t, x_{t}, z\right), g(\cdot)=g\left(t_{m}, x_{t_{m}}, z\right)$, i.e., the current values of the process $x_{t}$ are transmitted. Suppose that in this case the transmission is proceeded in an optimal manner.

Theorem 5. In the class $\mathcal{K}_{l}^{1}=\left\{\mathcal{H}_{l}^{1} ; \mathcal{G}_{l}^{1}\right\}$ of linear functionals

$$
\begin{aligned}
& \mathcal{H}_{l}^{1}=\left\{h(\cdot): h\left(t, x_{\tau}, z\right)=h(t, z)+H_{1}(t, z) x_{\tau}\right\}, \\
& \mathcal{G}_{l}^{1}=\left\{g(\cdot): g\left(t_{m}, x_{\tau}, z\right)=g\left(t_{m}, z\right)+G_{1}\left(t_{m}, z\right) x_{\tau}\right\}:
\end{aligned}
$$


$\left.1^{0}\right)$ optimal coding functionals $h^{0}\left(t, x_{\tau}, z^{0}\right), g^{0}\left(t_{m}, x_{\tau}, z^{0}\right)$ and optimal message $\left\{z_{t}^{0} ; \eta^{0}\left(t_{m}\right)\right\}$ are defined in the form

$$
\begin{aligned}
& h^{0}\left(t, z^{0}\right)=-H_{1}^{0}\left(t, z^{0}\right) \mu^{0}(\tau, t), \quad H_{1}^{0}\left(t, z^{0}\right)=\left[\tilde{h}(t) / \Delta_{11}^{0}(\tau, t)\right]^{1 / 2} \\
& g^{0}\left(t_{m}, z^{0}\right)=-G_{1}^{0}\left(t_{m}, z^{0}\right) \mu^{0}\left(\tau, t_{m}-0\right) \\
& G_{1}^{0}\left(t_{m}, z^{0}\right)=\left[\tilde{g}\left(t_{m}\right) / \Delta_{11}^{0}\left(\tau, t_{m}-0\right)\right]^{1 / 2} \\
& \mathrm{~d} z_{t}^{0}=\left[\tilde{h}(t) / \Delta_{11}^{0}(\tau, t)\right]^{1 / 2}\left[x_{\tau}-\mu^{0}(\tau, t)\right] \mathrm{d} t+\Phi_{2}(t) \mathrm{d} v_{t} \\
& \eta^{0}\left(t_{m}\right)=\left[\tilde{g}\left(t_{m}\right) / \Delta_{11}^{0}\left(\tau, t_{m}-0\right)\right]^{1 / 2}\left[x_{\tau}-\mu^{0}\left(\tau, t_{m}-0\right)\right]+\Phi_{3}\left(t_{m}\right) \xi\left(t_{m}\right)
\end{aligned}
$$

$\left.2^{0}\right)$ optimal decoding $\mu^{0}(t)$ and a minimal decoding error $\Delta^{0}(t)$ on the intervals $t_{m} \leqslant t<t_{m+1}$ are defined by the equations

$$
\begin{gathered}
\mathrm{d} \mu^{0}(t)=F(t) \mu^{0}(t) \mathrm{d} t+R^{-1}(t)\left[\tilde{h}(t) / \Delta_{11}^{0}(\tau, t)\right]^{1 / 2} \Delta_{01}^{0}(\tau, t) \mathrm{d} z_{t}^{0} \\
\mathrm{~d} \Delta^{0}(t) / \mathrm{d} t=\left(2 F(t)-R^{-1}(t) \tilde{h}(t)\left[\left(\Delta_{01}^{0}(\tau, t)\right)^{2} / \Delta^{0}(t) \Delta_{11}^{0}(\tau, t)\right]\right) \Delta^{0}(t) \\
+Q(t)
\end{gathered}
$$

with the initial conditions

$$
\begin{aligned}
& \mu^{0}\left(t_{m}\right)=\mu^{0}\left(t_{m}-0\right) \\
& +\Delta_{01}^{0}\left(\tau, t_{m}-0\right)\left[\tilde{g}\left(t_{m}\right) / \Delta_{11}^{0}\left(\tau, t_{m}-0\right)\right]^{1 / 2}\left[V\left(t_{m}\right)+\tilde{g}\left(t_{m}\right)\right]^{-1} \eta^{0}\left(t_{m}\right), \\
& \Delta^{0}\left(t_{m}\right)=\Delta^{0}\left(t_{m}-0\right) \frac{V\left(t_{m}\right)}{\left[V\left(t_{m}\right)+\tilde{g}\left(t_{m}\right)\right]} \\
& \times\left[1+\frac{\tilde{g}\left(t_{m}\right)}{V\left(t_{m}\right)}\left(1-\frac{\left(\Delta_{01}^{0}\left(\tau, t_{m}-0\right)\right)^{2}}{\Delta^{0}\left(t_{m}-0\right) \Delta_{11}^{0}\left(\tau, t_{m}-0\right)}\right)\right],
\end{aligned}
$$

where $Q(t)=\Phi_{1}^{2}(t), R(t)=\Phi_{2}^{2}(t), V\left(t_{m}\right)=\Phi_{3}^{2}\left(t_{m}\right)$;

$\left.\left.3^{0}\right) \mu^{0}(\tau, t)=M\left\{x_{\tau} \mid\left(z^{0}\right)_{0}^{t},\left(\eta^{0}\right)_{0}^{m}\right\}, \Delta_{11}^{0}(\tau, t)=M\left\{\left[x_{\tau}-\mu^{0}(\tau, t)\right]^{2}\right\}\right)$, and $\left.\Delta_{01}^{0}(\tau, t)==M\left\{\left[x_{t}-\mu^{0}(t)\right]\left[x_{\tau}-\mu^{0}(\tau, t)\right]\right\}\right)$ on the intervals $t_{m} \leqslant t<t_{m+1}$ are defined by the equations

$$
\begin{aligned}
& \mathrm{d} \mu^{0}(\tau, t)=R^{-1}(t)\left[\tilde{h}(t) \Delta_{11}^{0}(\tau, t)\right]^{1 / 2} \mathrm{~d} z_{t}^{0}, \\
& \mathrm{~d} \Delta_{11}^{0}(\tau, t) / \mathrm{d} t=-R^{-1}(t) \tilde{h}(t) \Delta_{11}^{0}(\tau, t), \\
& \mathrm{d} \Delta_{01}^{0}(\tau, t) / \mathrm{d} t=\left[F(t)-R^{-1}(t) \tilde{h}(t)\right] \Delta_{01}^{0}(\tau, t)
\end{aligned}
$$

with the initial conditions

$$
\begin{aligned}
& \mu^{0}\left(\tau, t_{m}\right)=\mu^{0}\left(\tau, t_{m}-0\right) \\
& \quad+\left[\tilde{g}\left(t_{m}\right) \Delta_{11}^{0}\left(\tau, t_{m}-0\right)\right]^{1 / 2}\left[V\left(t_{m}\right)+\tilde{g}\left(t_{m}\right)\right]^{-1} \eta^{0}\left(t_{m}\right), \\
& \Delta_{11}^{0}\left(\tau, t_{m}\right)=V\left(t_{m}\right)\left[V\left(t_{m}\right)+\tilde{g}\left(t_{m}\right)\right]^{-1} \Delta_{11}^{0}\left(\tau, t_{m}-0\right), \\
& \Delta_{01}^{0}\left(\tau, t_{m}\right)=V\left(t_{m}\right)\left[V\left(t_{m}\right)+\tilde{g}\left(t_{m}\right)\right]^{-1} \Delta_{01}^{0}\left(\tau, t_{m}-0\right) .
\end{aligned}
$$


Proof. If given $\{h(\cdot) ; g(\cdot)\} \in \mathcal{K}_{l}^{1}$ on the intervals $t_{m} \leqslant t<t_{m+1}$ (see (Abakumova et al., 1995b; Dyomin et al., 1997) and Proposition $\left.2^{*}\right) \mu(t)$ and $\gamma(t)$ are defined by the equations

$$
\begin{aligned}
& \mathrm{d} \mu(t)=F(t) \mu(t) \mathrm{d} t+R^{-1}(t) H_{1}(t, z) \gamma_{01}(\tau, t) \mathrm{d} \tilde{z}_{t}, \\
& \mathrm{~d} \gamma(t) / \mathrm{d} t=2 F(t) \gamma(t)-R^{-1}(t) H_{1}^{2}(t, z) \gamma_{01}^{2}(\tau, t)+Q(t)
\end{aligned}
$$

with the initial conditions

$$
\begin{aligned}
\mu\left(t_{m}\right)= & \mu\left(t_{m}-0\right)+G_{1}\left(t_{m}, z\right) \gamma_{01}\left(\tau, t_{m}-0\right)\left[V\left(t_{m}\right)\right. \\
& \left.+G_{1}^{2}\left(t_{m}, z\right) \gamma_{11}\left(\tau, t_{m}-0\right)\right]^{-1} \tilde{\eta}\left(t_{m}\right), \\
\gamma\left(t_{m}\right)= & \gamma\left(t_{m}-0\right)-G_{1}^{2}\left(t_{m}, z\right) \gamma_{01}^{2}\left(\tau, t_{m}-0\right)\left[V\left(t_{m}\right)\right. \\
& \left.+G_{1}^{2}\left(t_{m}, z\right) \gamma_{11}\left(\tau, t_{m}-0\right)\right]^{-1},
\end{aligned}
$$

where $\mu(\tau, t)=M\left\{x_{\tau} \mid z_{0}^{t}, \eta_{0}^{m}\right\}, \gamma_{01}(\tau, t)=M\left\{\left[x_{t}-\mu(t)\right]\left[x_{\tau}-\mu(\tau, t)\right] \mid z_{0}^{t}, \eta_{0}^{m}\right\}$, $\gamma_{11}(\tau, t)=M\left\{\left[x_{\tau}-\mu(\tau, t)\right]^{2} \mid z_{0}^{t}, \eta_{0}^{m}\right\}$,

$$
\begin{aligned}
& \mathrm{d} \tilde{z}_{t}=\mathrm{d} z_{t}-\left[h(t, z)+H_{1}(t, z) \mu(\tau, t)\right] \mathrm{d} t, \\
& \tilde{\eta}\left(t_{m}\right)=\eta\left(t_{m}\right)-\left[g\left(t_{m}, z\right)+G_{1}\left(t_{m}, z\right) \mu\left(\tau, t_{m}-0\right)\right] .
\end{aligned}
$$

Since $M\{\cdot\}=M\left\{M\left\{\cdot \mid z_{0}^{t_{m}}, \eta_{0}^{m-1}\right\}\right\}$, the use of (5.4) in (5.3) yields

$$
\begin{aligned}
M\left\{g^{2}(\cdot)\right\}= & M\left\{\left[g\left(t_{m}, z\right)+G_{1}\left(t_{m}, z\right) \mu\left(\tau, t_{m}-0\right)\right]^{2}\right\} \\
& +M\left\{G_{1}^{2}\left(t_{m}, z\right) \gamma_{11}\left(\tau, t_{m}-0\right)\right\} \leqslant \tilde{g}\left(t_{m}\right) .
\end{aligned}
$$

Formula (5.22) can be presented in the form

$$
\begin{aligned}
\gamma\left(t_{m}\right)= & \gamma\left(t_{m}-0\right)-\frac{\gamma_{01}^{2}\left(\tau, t_{m}-0\right)}{\gamma_{11}\left(\tau, t_{m}-0\right)} \\
& +\frac{\gamma_{01}^{2}\left(\tau, t_{m}-0\right)}{\gamma_{11}\left(\tau, t_{m}-0\right)} \frac{V\left(t_{m}\right)}{\left[V\left(t_{m}\right)+G_{1}^{2}\left(t_{m}, z\right) \gamma_{11}\left(\tau, t_{m}-0\right)\right]} .
\end{aligned}
$$

Suppose that for $t<t_{m}$ the transmission was proceeded in an optimal manner. Then, $\gamma\left(t_{m}-0\right), \gamma_{11}\left(\tau, t_{m}-0\right)$ and $\gamma_{01}\left(\tau, t_{m}-0\right)$ are replaced by $\Delta^{0}\left(t_{m}-0\right), \Delta_{11}^{0}\left(\tau, t_{m}-0\right)$, $\Delta_{01}^{0}\left(\tau, t_{m}-0\right)$ which are nonrandom. From (5.24), (5.25) for $\Delta\left(t_{m}\right)=M\left\{\gamma\left(t_{m}\right)\right\}$ taking into account Jensen inequality $M\left\{Y^{-1}\right\} \geqslant(M\{Y\})^{-1}$ (Liptser and Shiryayev, 1977 ; 1978) it follows that

$$
\begin{aligned}
\Delta\left(t_{m}\right) \geqslant & \Delta^{0}\left(t_{m}-0\right)+\frac{\left(\Delta_{01}^{0}\left(\tau, t_{m}-0\right)\right)^{2}}{\Delta_{11}^{0}\left(\tau, t_{m}-0\right)} \\
& +\frac{\left(\Delta_{01}^{0}\left(\tau, t_{m}-0\right)\right)^{2}}{\Delta_{11}^{0}\left(\tau, t_{m}-0\right)} \frac{V\left(t_{m}\right)}{\left[V\left(t_{m}\right)+\tilde{g}\left(t_{m}\right)\right]}
\end{aligned}
$$




$$
\begin{aligned}
= & \Delta^{0}\left(t_{m}-0\right) \frac{V\left(t_{m}\right)}{\left[V\left(t_{m}\right)+\tilde{g}\left(t_{m}\right)\right]} \\
& \times\left[1+\frac{\tilde{g}\left(t_{m}\right)}{V\left(t_{m}\right)}\left(1-\frac{\left(\Delta_{01}^{0}\left(\tau, t_{m}-0\right)\right)^{2}}{\Delta^{0}\left(t_{m}-0\right) \Delta_{11}^{0}\left(\tau, t_{m}-0\right)}\right)\right] .
\end{aligned}
$$

The use of (5.6) in (5.25) yields that $\gamma^{0}\left(t_{m}\right)=\Delta^{0}\left(t_{m}\right)$ which is defined by (5.12). It follows from (5.12) and (5.26) that coincidence $\gamma^{0}\left(t_{m}\right)$ with the low bound for $\Delta\left(t_{m}\right)$ proves an optimality of the coding (5.6) and (5.8), (5.11), (5.12) follows as a result of substitution (5.26) into (5.2), (5.21), (5.22) taking into account (5.23). If given $\{h(\cdot) ; g(\cdot)\} \in \mathcal{K}_{l}^{1}$ on the intervals $t_{m} \leqslant t<t_{m+1}$ (see (Abakumova et al., 1995b; Dyomin et al., 1997) and Proposition $\left.2^{*}\right) \mu(\tau, t), \gamma_{11}(\tau, t)$ and $\gamma_{01}(\tau, t)$ are defined by the equations

$$
\begin{aligned}
& \mathrm{d} \mu(\tau, t)=R^{-1}(t) H_{1}(t, z) \gamma_{11}(\tau, t) \mathrm{d} \tilde{z}_{t}, \\
& \mathrm{~d} \gamma_{11}(\tau, t) / \mathrm{d} t=-R^{-1}(t) H_{1}^{2}(t, z) \gamma_{11}^{2}(\tau, t), \\
& \mathrm{d} \gamma_{01}(\tau, t) / \mathrm{d} t=\left[F(t)-R^{-1}(t) H_{1}^{2}(t, z) \gamma_{11}(\tau, t)\right] \gamma_{01}(\tau, t)
\end{aligned}
$$

with the initial conditions

$$
\begin{aligned}
& \mu\left(\tau, t_{m}\right)=\mu\left(\tau, t_{m}-0\right) \\
& \quad+G_{1}\left(t_{m}, z\right) \gamma_{11}\left(\tau, t_{m}-0\right)\left[V\left(t_{m}\right)+G_{1}^{2}\left(t_{m}, z\right) \gamma_{11}\left(\tau, t_{m}-0\right)\right]^{-1} \tilde{\eta}\left(t_{m}\right) \\
& \gamma_{11}\left(\tau, t_{m}\right)=\gamma_{11}\left(\tau, t_{m}-0\right) \\
& \quad-G_{1}^{2}\left(t_{m}, z\right) \gamma_{11}^{2}\left(\tau, t_{m}-0\right)\left[V\left(t_{m}\right)+G_{1}^{2}\left(t_{m}, z\right) \gamma_{11}\left(\tau, t_{m}-0\right)\right]^{-1} \\
& \gamma_{01}\left(\tau, t_{m}\right)=\gamma_{01}\left(\tau, t_{m}-0\right) \\
& \quad-G_{1}^{2}\left(t_{m}, z\right) \gamma_{01}\left(\tau, t_{m}-0\right) \gamma_{11}\left(\tau, t_{m}-0\right)\left[V\left(t_{m}\right)+G_{1}^{2}\left(t_{m}, z\right) \gamma_{11}\left(\tau, t_{m}-0\right)\right]^{-1}(5 .
\end{aligned}
$$

The substitution of (5.6) into (5.30)-(5.32), taking into account (5.23), gives (5.16)(5.18).

Let us represent the (5.20) in the form

$$
\begin{aligned}
\mathrm{d} \gamma(t) / \mathrm{d} t=( & 2 F(t)-R^{-1}(t) H_{1}^{2}(t, z) \gamma_{11}(\tau, t) \\
& \left.\times\left[\gamma_{01}^{2}(\tau, t) / \gamma(t) \gamma_{11}(\tau, t)\right]\right) \gamma(t)+Q(t) .
\end{aligned}
$$

Suppose that up to the moment $t \leqslant t_{m}$ the transmission is proceeded in an optimal manner. Then differential equation (5.33) on the time interval $t_{m} \leqslant t<t_{m+1}$ is equivalent to integral equation

$$
\begin{aligned}
& \gamma(t)=\Delta^{0}\left(t_{m}\right) \\
& \quad \times \exp \left\{\int_{t_{m}}^{t}\left(2 F(\sigma)-R^{-1}(\sigma) H_{1}^{2}(\sigma, z) \gamma_{11}(\tau, \sigma)\left[\gamma_{01}^{2}(\tau, \sigma) / \gamma(\sigma) \gamma_{11}(\tau, \sigma)\right]\right) \mathrm{d} \sigma\right\}
\end{aligned}
$$




$$
\begin{aligned}
+\int_{t_{m}}^{t} Q(\sigma) \exp \left\{\int_{\sigma}^{t}\right. & \left(2 F(u)-R^{-1}(u) H_{1}^{2}(u, z) \gamma_{11}(\tau, u)\right. \\
& \left.\left.\times\left[\gamma_{01}^{2}(\tau, u) / \gamma(u) \gamma_{11}(\tau, u)\right]\right) \mathrm{d} u\right\} \mathrm{d} \sigma
\end{aligned}
$$

validity of which is proved by differentiating with respect to $t$. Since $M\{\cdot\}=$ $M\left\{M\left\{\cdot \mid z_{0}^{t}, \eta_{0}^{m}\right\}\right\}$, then the use of (5.4) in (5.3) yields

$$
\begin{aligned}
M\left\{h^{2}(\cdot)\right\}= & M\left\{\left[h(t, z)+H_{1}(t, z) \mu(\tau, t)\right]^{2}\right\} \\
& +M\left\{H_{1}^{2}(t, z) \gamma_{11}(\tau, t)\right\} \leqslant \tilde{h}(t) .
\end{aligned}
$$

Using of Jensen inequality $M\{\varphi(Y)\} \geqslant \varphi(M\{Y\})$ (Liptser and Shiryayev, 1977; 1978) for the convex function $\varphi(y)=\exp \{y\}$ in (5.34), we obtain inequality for $\Delta(t)=$ $M\{\gamma(t)\}$

$$
\begin{gathered}
\Delta(t) \geqslant \Delta^{0}\left(t_{m}\right) \exp \left\{\int _ { t _ { m } } ^ { t } \left[2 F(\sigma)-R^{-1}(\sigma) M\left\{H_{1}^{2}(\sigma, z) \gamma_{11}(\tau, \sigma)\right.\right.\right. \\
\left.\left.\times\left[\gamma_{01}^{2}(\tau, \sigma) / \gamma(\sigma) \gamma_{11}(\tau, \sigma)\right]\right\} \mathrm{d} \sigma\right\} \\
+\int_{t_{m}} Q(\sigma) \exp \left\{\int _ { \sigma } ^ { t } \left(2 F(u)-R^{-1}(u) M\left\{H_{1}^{2}(u, z) \gamma_{11}(\tau, u)\right.\right.\right. \\
\left.\left.\left.\times\left[\gamma_{01}^{2}(\tau, u) / \gamma(u) \gamma_{11}(\tau, u)\right]\right\}\right) \mathrm{d} u\right\} \mathrm{d} \sigma .
\end{gathered}
$$

The use of (5.5) in (5.20), (5.28), (5.29) brings us to equations for $\gamma^{0}(t), \gamma_{11}^{0}(\tau, t)$, $\gamma_{01}^{0}(\tau, t)$ on the interval $t_{m} \leqslant t<t_{m+1}$

$$
\begin{aligned}
& \mathrm{d} \gamma^{0}(t) / \mathrm{d} t=\left(2 F(t)-R^{-1}(t) \tilde{h}(t)\left[\left(\gamma_{01}^{0}(\tau, t)\right)^{2} / \gamma^{0}(t) \Delta_{11}^{0}(\tau, t)\right]\right) \gamma^{0}(t)+Q(t),(5) \\
& \gamma^{0}\left(t_{m}\right)=\Delta^{0}\left(t_{m}\right) \\
& \mathrm{d} \gamma_{11}^{0}(\tau, t) / \mathrm{d} t=-R^{-1}(t) \tilde{h}(t)\left[\left(\gamma_{11}^{0}(\tau, t)\right)^{2} / \Delta_{11}^{0}(\tau, t)\right] \\
& \gamma_{11}^{0}\left(\tau, t_{m}\right)=\Delta_{11}^{0}\left(\tau, t_{m}\right) \\
& \mathrm{d} \gamma_{01}^{0}(\tau, t) / \mathrm{d} t=\left(F(t)-R^{-1}(t) \tilde{h}(t)\left[\gamma_{11}^{0}(\tau, t) / \Delta_{11}^{0}(\tau, t)\right]\right) \gamma_{01}^{0}(\tau, t) \\
& \gamma_{01}^{0}\left(\tau, t_{m}\right)=\Delta_{01}^{0}\left(\tau, t_{m}\right)
\end{aligned}
$$

Equations (5.37)-(5.39) imply that $\gamma^{0}(t), \gamma_{01}^{0}(\tau, t)$, and $\gamma_{11}^{0}(\tau, t)$ are nonrandom values. According to (5.35), we have $M\left\{H_{1}^{2}(t, z)\right\} \gamma_{11}^{0}(\tau, t) \leqslant \tilde{h}(t)$. Then, from (5.36), we ob- 
tain

$$
\begin{aligned}
\Delta(t) \geqslant \Delta^{0}\left(t_{m}\right) & \exp \left\{\int _ { t _ { m } } ^ { t } \left[2 F(\sigma)-R^{-1}(\sigma) \tilde{h}(\sigma)\right.\right. \\
& \left.\left.\times\left[\left(\gamma_{01}^{0}(\tau, \sigma)\right)^{2} / \gamma^{0}(\sigma) \gamma_{11}^{0}(\tau, \sigma)\right]\right) \mathrm{d} \sigma\right\} \\
+ & \int_{t_{m}}^{t} Q(\sigma) \exp \left\{\int _ { \sigma } ^ { t } \left(2 F(u)-R^{-1}(u) \tilde{h}(u)\right.\right. \\
& \left.\left.\times\left[\left(\gamma_{01}^{0}(\tau, u)\right)^{2} / \gamma^{0}(u) \gamma_{11}^{0}(\tau, u)\right]\right) \mathrm{d} u\right\} \mathrm{d} \sigma .
\end{aligned}
$$

Suppose $\Delta^{0}(t)$ is the right part of (5.40). Then, differentiation with respect to $t$ gives that $\Delta^{0}(t)$ is defined by the equation

$$
\begin{aligned}
& \mathrm{d} \Delta^{0}(t) / \mathrm{d} t=\left(2 F(t)-R^{-1}(t) \tilde{h}(t)\left[\left(\gamma_{01}^{0}(\tau, t)\right)^{2} / \gamma^{0}(t) \gamma_{11}^{0}(\tau, t)\right]\right) \Delta^{0}(t)+Q(t),(5.41) \\
& \left.\Delta^{0}(t)\right|_{t=t_{m}}=\Delta^{0}\left(t_{m}\right) .
\end{aligned}
$$

It follows from (5.14), (5.38) and (5.15), (5.39) that the solutions of these equations are coincident, i.e., $\gamma_{11}^{0}(\tau, t)=\Delta_{11}^{0}(\tau, t), \gamma_{01}^{0}(\tau, t)=\Delta_{01}^{0}(\tau, t)$. Therefore, the solutions of (5.37) and (5.41), (5.10) are coincident, i.e., $\gamma^{0}(t)=\Delta^{0}(t)$. Coincidence $\gamma^{0}(t)$ with the low bound for $\Delta(t)$ proves an optimality of coding (5.5). Equations (5.7), (5.9), (5.13) follow as a result of substitution (5.5) in (5.2), (5.19), (5.27). The validity of proved result for arbitrary time interval $\tau \leqslant t_{m} \leqslant t<t_{m+1}$ is derived with respect to induction, taking into account Remark 5.

Theorem 6. Suppose $I_{t}^{0}\left[x_{t} ;\left(z^{0}\right)_{0}^{t},\left(\eta^{0}\right)_{0}^{m}\right]$ is the information amount, attained on the coding functionals (5.5), (5.6). Then property

$$
I_{t}^{0}\left[x_{t} ;\left(z^{0}\right)_{0}^{t},\left(\eta^{0}\right)_{0}^{m}\right]=\sup I_{t}\left[x_{t} ; z_{0}^{t}, \eta_{0}^{m}\right]
$$

takes place, where the supremum is taken for $\{h(\cdot) ; g(\cdot)\} \in \mathcal{K}_{l}^{1}$, and

$$
\begin{aligned}
& I_{t}^{0}\left[x_{t} ;\left(z^{0}\right)_{0}^{t},\left(\eta^{0}\right)_{0}^{m}\right] \\
& \quad+\frac{1}{2} \sum_{\tau \leqslant t_{i} \leqslant t} \ln \left(\left[1+\frac{\tilde{g}\left(t_{i}\right)}{V\left(t_{i}\right)}\right]\left[1+\frac{\tilde{g}\left(t_{i}\right)}{V\left(t_{i}\right)}\left(1-\frac{\left(\Delta_{01}^{0}\left(\tau, t_{i}-0\right)\right)^{2}}{\Delta^{0}\left(t_{i}-0\right) \Delta_{11}^{0}\left(\tau, t_{i}-0\right)}\right)\right]^{-1}\right) \\
& \quad+\frac{1}{2} \int_{\tau}^{t}\left[\frac{\tilde{h}(\sigma)}{R(\sigma)} \frac{\left(\Delta_{01}^{0}(\tau, \sigma)\right)^{2}}{\Delta^{0}(\sigma) \Delta_{11}^{0}(\tau, t)}-Q(\sigma)\left(\frac{1}{\Delta^{0}(\sigma)}-\frac{1}{D(\sigma)}\right)\right] \mathrm{d} \sigma
\end{aligned}
$$


Proof. According to Ihara inequality (Lipser, 1974; Lipser and Shiryayev, 1977; 1978) $\Delta(t) \geqslant D(t) \exp \left\{-2 I_{t}[\cdot]\right\}$, we obtain

$$
I_{t}[\cdot] \leqslant(1 / 2) \ln [D(t) / \Delta(t)]
$$

Since, in accordance with Theorem $5 \inf \Delta(t)=\Delta^{0}(t)$, then from (5.44), we have

$$
\sup I_{t}[\cdot]=I_{t}^{0}\left[x_{t} ;\left(z^{0}\right)_{0}^{t},\left(\eta^{0}\right)_{0}^{m}\right]=(1 / 2) \ln \left[D(t) / \Delta^{0}(t)\right]
$$

Since on coding (5.5) and (5.6) $p_{t}(x)=\mathcal{N}\left\{x ; \mu^{0}(t), \Delta^{0}(t)\right\}$ and $p(t, x)=$ $\mathcal{N}\{x ; a(t), D(t)\}$, we obtain

$$
I_{t}^{0}[\cdot]=M\left\{\ln \left[p_{t}\left(x_{t}\right) / p\left(t, x_{t}\right)\right]\right\}=(1 / 2) \ln \left[D(t) / \Delta^{0}(t)\right]
$$

Coincidence (5.45) with (5.46) proves the property (5.42). From (5.46) follows that

$$
\frac{\mathrm{d} I_{t}^{0}[\cdot]}{\mathrm{d} t}=\frac{1}{2}\left[\frac{1}{D(t)} \frac{\mathrm{d} D(t)}{\mathrm{d} t}-\frac{1}{\Delta^{0}(t)} \frac{\mathrm{d} \Delta^{0}(t)}{\mathrm{d} t}\right]
$$

Since for Gaussian process $x_{t}$, defined by the equation (5.1), we have

$$
d D(t) / \mathrm{d} t=2 F(t) D(t)+Q(t)
$$

then, the use of (5.10), (5.48) in (5.47) for $t_{m} \leqslant t<t_{m+1}$ gives

$$
\frac{\mathrm{d} I_{t}^{0}[\cdot]}{\mathrm{d} t}=\frac{1}{2}\left(\frac{\tilde{h}(t)}{R(t)} \frac{\left(\Delta_{01}^{0}(\tau, t)^{2}\right.}{\Delta^{0}(t) \Delta_{11}^{0}(\tau, t)}-Q(t)\left[\frac{1}{\Delta^{0}(t)}-\frac{1}{D(t)}\right]\right) .
$$

From (5.46) follows that

$$
I_{t_{m}}^{0}[\cdot]=(1 / 2) \ln \left[D\left(t_{m}\right) / \Delta^{0}\left(t_{m}\right)\right]
$$

Using (5.12) in (5.50), we obtain

$$
\begin{aligned}
& I_{t_{m}}^{0}[\cdot]=I_{t_{m}-0}^{0}[\cdot] \\
& \quad+\frac{1}{2} \ln \left(\left[1+\frac{\tilde{g}\left(t_{m}\right)}{V\left(t_{m}\right)}\right]\left[1+\frac{\tilde{g}\left(t_{m}\right)}{V\left(t_{m}\right)}\left(1-\frac{\left(\Delta_{01}^{0}\left(\tau, t_{m}-0\right)\right)^{2}}{\Delta^{0}\left(t_{m}-0\right) \Delta_{11}^{0}\left(\tau, t_{m}-0\right)}\right)\right]^{-1}\right)
\end{aligned}
$$

Formula (5.43) obviously follows from (5.49), (5.51).

COROllary 3. The solution of optimal transmission problem on the interval $0 \leqslant t_{0} \leqslant$ $t_{m} \leqslant t<\tau$ is defined by Theorem $5^{*}$ and $I_{t}^{0}\left[x_{t},\left(z^{0}\right)_{0}^{t},\left(\eta^{0}\right)_{0}^{m}\right]$ is determined by Theorem $7^{*}$.

Correctness of this result follows from Remark 5 and Remark $5^{*}$. 
REMARK 6. The usage of (5.4)-(5.6) in (5.3) gives that $M\left\{\left[h^{0}\left(t, x_{\tau}, z^{0}\right)\right]^{2}\right\}=\tilde{h}(t)$, $M\left\{\left[g^{0}\left(t_{m}, x_{\tau}, z^{0}\right)\right]^{2}\right\}=\tilde{g}\left(t_{m}\right)$, i.e., by the optimal means of transmission energy capacities of continuous and discrete transmission channels are completely applied.

REMARK 7. Since capacity $C[0, T]$ of the transmission channel is defined in the form of $C[0, T]=\sup \left\{(1 / T) I_{T}[\cdot]\right\}$ (Shannon and Weaver, 1949; Gallager, 1968), according to Theorem 6 by continuous-discrete way of transmission (5.1)-(5.3) the coding functionals (5.5), (5.6) provide the transmission of a maximum possible information amount in the class $\mathcal{K}_{l}^{1}$ of the linear functionals (5.4).

REMARK 8 . The proof of the similar Theorem $6^{*}$ result is open for research.

Let us consider the cases: 1) the continuous channel with memory and the discrete channel with lag, i.e., $h(\cdot)=h\left(t, x_{t}, x_{\tau}, z\right), g(\cdot)=g\left(t_{m}, x_{\tau}, z\right)$; 2) the continuous channel with lag and the discrete channel with memory, i.e., $h(\cdot)=h\left(t, x_{\tau}, z\right), g(\cdot)=$ $g\left(t_{m}, x_{t_{m}}, x_{\tau}, z\right)$.

COROLlaRY 4. In the class $\mathcal{K}_{l}^{0,1}=\left\{\mathcal{H}_{l} ; \mathcal{G}_{l}^{1}\right\}$ of linear functionals

$$
\begin{aligned}
& \mathcal{H}_{l}=\left\{h(\cdot): h\left(t, x_{t}, x_{\tau}, z\right)=h(t, z)+H_{0}(t, z) x_{t}+H_{1}(t, z) x_{\tau}\right\}, \\
& \mathcal{G}_{l}^{1}=\left\{g(\cdot): g\left(t_{m}, x_{\tau}, z\right)=g\left(t_{m}, z\right)+G_{1}\left(t_{m}, z\right) x_{\tau}\right\}:
\end{aligned}
$$

$\left.1^{0}\right)$ optimal coding functionals $h^{0}\left(t, x_{t}, x_{\tau}, z^{0}\right), g^{0}\left(t_{m}, x_{\tau}, z^{0}\right)$ have the form $(6.5)^{*}$ and (5.6);

$\left.2^{0}\right)$ optimal message $\left\{z_{t}^{0} ; \eta^{0}\left(t_{m}\right)\right\}$ are defined by formulae $(6.7)^{*}$ and (5.8);

$\left.3^{0}\right)$ optimal decoding $\mu^{0}(t)$ and a minimal decoding error $\Delta^{0}(t)$ on the intervals $t_{m} \leqslant$ $t<t_{m+1}$, are defined by $\left((6.9)^{*}\right)$ and $(6.10)^{*}$ with the initial conditions (5.11) and (5.12);

$\left.4^{0}\right) \mu^{0}(\tau, t), \Delta_{11}^{0}(\tau, t)$, and $\Delta_{01}^{0}(\tau, t)$ on the intervals $t_{m} \leqslant t<t_{m+1}$ are defined by the equations

$$
\begin{aligned}
& \mathrm{d}_{t} \mu^{0}(\tau, t)=R^{-1}(t)\left[\tilde{h}(t) / \Delta^{0}(t)\right]^{1 / 2} \Delta_{01}^{0}(\tau, t) \mathrm{d} z_{t}^{0}, \\
& \mathrm{~d} \Delta_{11}^{0}(\tau, t) / \mathrm{d} t=-R^{-1}(t)\left[\tilde{h}(t) / \Delta^{0}(t)\right]\left(\Delta_{01}^{0}(\tau, t)\right)^{2}
\end{aligned}
$$

and (5.15) with the initial conditions (5.16)-(5.18).

In the class $\mathcal{K}_{l}^{1,0}=\left\{\mathcal{H}_{l}^{1} ; \mathcal{G}_{l}\right\}$ of linear functionals

$$
\begin{aligned}
& \mathcal{H}_{l}^{1}=\left\{h(\cdot): h\left(t, x_{\tau}, z\right)=h(t, z)+H_{1}(t, z) x_{\tau}\right\} \\
& \mathcal{G}_{l}=\left\{g(\cdot): g\left(t_{m}, x_{t_{m}}, x_{\tau}, z\right)=g\left(t_{m}, z\right)+G_{0}\left(t_{m}, z\right) x_{t_{m}}+G_{1}\left(t_{m}, z\right) x_{\tau}\right\}:
\end{aligned}
$$

$\left.5^{0}\right)$ optimal coding functionals $h^{0}\left(t, x_{\tau}, z^{0}\right), g^{0}\left(t_{m}, x_{t_{m}}, x_{\tau}, z^{0}\right)$ have the form (5.5) and $(6.6)^{*}$;

$\left.6^{0}\right)$ optimal message $\left\{z_{t}^{0} ; \eta^{0}\left(t_{m}\right)\right\}$ are defined by formulae (5.7) and (6.8)*; 
$\left.7^{0}\right)$ optimal decoding $\mu^{0}(t)$ and a minimal decoding error $\Delta^{0}(t)$ on the intervals $t_{m} \leqslant$ $t<t_{m+1}$ are defined by (5.9) and 5.10 with the initial conditions $(6.11)^{*}$ and (6.12)*;

$\left.8^{0}\right) \mu^{0}(\tau, t), \Delta_{11}^{0}(\tau, t)$, and $\Delta_{01}^{0}(\tau, t)$ on the intervals $t_{m} \leqslant t<t_{m+1}$ are defined by (5.13)-(5.15) with the initial conditions

$$
\begin{aligned}
& \mu^{0}\left(\tau, t_{m}\right)=\mu^{0}\left(\tau, t_{m}-0\right) \\
& \quad+\Delta_{01}^{0}\left(\tau, t_{m}-0\right)\left[\tilde{g}\left(t_{m}\right) / \Delta^{0}\left(t_{m}-0\right)\right]^{1 / 2}\left[V\left(t_{m}\right)+\tilde{g}\left(t_{m}\right)\right]^{-1} \eta^{0}\left(t_{m}\right), \\
& \quad \Delta_{11}^{0}\left(\tau, t_{m}\right)=\Delta_{11}^{0}\left(\tau, t_{m}-0\right) \frac{V\left(t_{m}\right)}{\left[V\left(t_{m}\right)+\tilde{g}\left(t_{m}\right)\right]} \\
& \quad \times\left[1+\frac{\tilde{g}\left(t_{m}\right)}{V\left(t_{m}\right)}\left(1-\frac{\left(\Delta_{01}^{0}\left(\tau, t_{m}-0\right)\right)^{2}}{\Delta^{0}\left(t_{m}-0\right) \Delta_{11}^{0}\left(\tau, t_{m}-0\right)}\right)\right]
\end{aligned}
$$

for $\mu^{0}(\tau, t)$ and $\Delta_{11}^{0}\left(\tau, t_{m}\right)$, and with the initial condition (5.18) for $\Delta_{01}^{0}(\tau, t)$.

Proof. If given $\{h(\cdot) ; g(\cdot)\} \in \mathcal{K}_{l}$ of the form $(6.4)^{*}$ on the intervals $t_{m} \leqslant t<t_{m+1}$ (see (Abakumova et al., 1995b; Dyomin et al., 1997) and Proposition $\left.2^{*}\right) \mu(\tau, t) \gamma_{11}(\tau, t)$ and $\gamma_{01}(\tau, t)$ are defined by the equations

$$
\begin{aligned}
& \mathrm{d} \mu(\tau, t)=R^{-1}(t)\left[H_{0}(t, z) \gamma_{01}(\tau, t)+H_{1}(t, z) \gamma_{11}(\tau, t)\right] \mathrm{d} \tilde{z}_{t}, \\
& \mathrm{~d} \gamma_{11}(\tau, t) / \mathrm{d} t=-R^{-1}(t)\left[H_{0}(t, z) \gamma_{01}(\tau, t)+H_{1}(t, z) \gamma_{11}(\tau, t)\right]^{2}, \\
& \mathrm{~d} \gamma_{01}(\tau, t) / \mathrm{d} t=F(t) \gamma_{01}(\tau, t)-R^{-1}(t)\left[H_{0}(t, z) \gamma(t)+H_{1}(t, z) \gamma_{01}(\tau, t)\right] \\
& \quad \times\left[H_{0}(t, z) \gamma_{01}(\tau, t)+H_{1}(t, z) \gamma_{11}(\tau, t)\right]
\end{aligned}
$$

with initial conditions

$$
\begin{aligned}
& \mu\left(\tau, t_{m}\right)=\mu\left(\tau, t_{m}-0\right) \\
& \quad+\left[G_{0}\left(t_{m}, z\right) \gamma_{01}\left(\tau, t_{m}-0\right)+G_{1}\left(t_{m}, z\right) \gamma_{11}\left(\tau, t_{m}-0\right)\right] W^{-1}\left(t_{m}\right) \tilde{\eta}\left(t_{m}\right), \\
& \gamma_{11}\left(\tau, t_{m}\right)=\gamma_{11}\left(\tau, t_{m}-0\right) \\
& \quad-\left[G_{0}\left(t_{m}, z\right) \gamma_{01}\left(\tau, t_{m}-0\right)+G_{1}\left(t_{m}, z\right) \gamma_{11}\left(\tau, t_{m}-0\right)\right]^{2} W^{-1}\left(t_{m}\right), \\
& \gamma_{01}\left(\tau, t_{m}\right)=\gamma_{01}\left(\tau, t_{m}-0\right) \\
& \quad-\left[G_{0}\left(t_{m}, z\right) \gamma\left(t_{m}-0\right)+G_{1}\left(t_{m}, z\right) \gamma_{01}\left(\tau, t_{m}-0\right)\right] \\
& \quad \times\left[G_{0}\left(t_{m}, z\right) \gamma_{01}\left(\tau, t_{m}-0\right)+G_{1}\left(t_{m}, z\right) \gamma_{11}\left(\tau, t_{m}-0\right)\right] W^{-1}\left(t_{m}\right),
\end{aligned}
$$

where $\mathrm{d} \tilde{z}_{t}=\mathrm{d} z_{t}-\left[h(t, z)+H_{0}(t, z) \mu(t)+H_{1}(t, z) \mu(\tau, t)\right] \mathrm{d} t, \tilde{\eta}\left(t_{m}\right)=\eta\left(t_{m}\right)-$ $\left[g\left(t_{m}, z\right)+G_{0}\left(t_{m}, z\right) \mu\left(t_{m}-0\right)+G_{1}\left(t_{m}, z\right) \mu\left(\tau, t_{m}-0\right)\right]$ and $W\left(t_{m}\right)$ are determined by formula $(6.17)^{*}$. Equations (5.53), (5.54), and (5.15) are obtained as a result of using $(6.5)^{*}$ in (5.58)-(5.60). Initial conditions (5.56), (5.57), (5.18) are obtained as a result of using $(6.6)^{*}$ in (5.61)-(5.63). The other statements of Corollary obviously follow from Theorem 5 and Theorem $5^{*}$. 
COROLlary 5. $\left.1^{0}\right)$ In the class $\mathcal{K}_{l}^{0,1}=\left\{\mathcal{H}_{l} ; \mathcal{G}_{l}^{1}\right\}$ of the form (5.52) the property (5.42) takes place and

$$
\begin{aligned}
& I_{t}^{0}\left[x_{t} ;\left(z^{0}\right)_{0}^{t},\left(\eta^{0}\right)_{0}^{m}\right] \\
& =\frac{1}{2} \sum_{\tau \leqslant t_{i} \leqslant t} \ln \left(\left[1+\frac{\tilde{g}\left(t_{i}\right)}{V\left(t_{i}\right)}\right]\left[1+\frac{\tilde{g}\left(t_{i}\right)}{V\left(t_{i}\right)}\left(1-\frac{\left(\Delta_{01}^{0}\left(\tau, t_{i}-0\right)\right)^{2}}{\Delta^{0}\left(t_{i}-0\right) \Delta_{11}^{0}\left(\tau, t_{i}-0\right)}\right)\right]^{-1}\right) \\
& \quad+\frac{1}{2} \int_{\tau}^{t}\left(R^{-1}(\sigma) \tilde{h}(\sigma)-Q(\sigma)\left[\left(\Delta^{0}(\sigma)\right)^{-1}-D^{-1}(\sigma)\right]\right) \mathrm{d} \sigma .
\end{aligned}
$$

$\left.2^{0}\right)$ In the class $\mathcal{K}_{l}^{1,0}=\left\{\mathcal{H}_{l}^{1} ; \mathcal{G}_{l}\right\}$ of the form (5.55) the property (5.42) takes place and

$$
\begin{aligned}
& I_{t}^{0}\left[x_{t} ;\left(z^{0}\right)_{0}^{t},\left(\eta^{0}\right)_{0}^{m}\right]=(1 / 2) \sum_{\tau \leqslant t_{i} \leqslant t} \ln \left[1+\left(\tilde{g}\left(t_{i}\right) / V\left(t_{i}\right)\right)\right] \\
& \quad+\frac{1}{2} \int_{\tau}^{t}\left[\frac{\tilde{h}(\sigma)}{R(\sigma)} \frac{\left(\Delta_{01}^{0}(\tau, \sigma)\right)^{2}}{\Delta^{0}(\sigma) \Delta_{11}^{0}(\tau, \sigma)}-Q(\sigma)\left(\frac{1}{\Delta^{0}(\sigma)}-\frac{1}{D(\sigma)}\right)\right] \mathrm{d} \sigma .
\end{aligned}
$$

Proof. For $t_{m} \leqslant t<t_{m+1}$ using $(6.10)^{*}$ and (5.48) in (5.47), we obtain

$$
\mathrm{d} I_{t}^{0}[\cdot] / \mathrm{d} t=(1 / 2)\left(R^{-1}(t) \tilde{h}(t)-Q(t)\left[\left(\Delta^{0}(t)\right)^{-1}-D^{-1}(t)\right]\right) .
$$

Then, (5.64) arises from (5.51), (5.66). The use of $(6.12)^{*}$ in (5.50) gives

$$
I_{t_{m}}^{0}[\cdot]=I_{t_{m}-0}^{0}[\cdot]+(1 / 2) \ln \left[1+\left(\tilde{g}\left(t_{m}\right) / V\left(t_{m}\right)\right)\right] .
$$

Then, (5.65) arises from (5.49), (5.67).

Assume that a continuous channel is the transmission channel with memory, i.e., $h(\cdot) \in \mathcal{H}_{l}$. Let us consider the efficiency of discrete transmission with memory in relation to discrete transmission with lag under optimal method of transmission, denoting the mean square errors reproduction of signal for these two cases by $\Delta^{0}\left(t_{m}\right)$ and $\widetilde{\Delta}^{0}\left(t_{m}\right)$, respectively. As the efficiency measure taken the value $\varepsilon_{f}(t)=\Delta^{0}(t) / \widetilde{\Delta}^{0}(t)$.

REMARK 9. Since for case optimal method of transmission under the energy limitations $(6.3)^{*}$ in the channels with memory only current values of the process $x_{t}$ are transmitted, then according to Remark $5^{*}$ channels with memory and without memory are equivalent.

Let us denote

$$
\begin{aligned}
& t^{*}=t-\tau, \quad \delta_{c}(t)=\tilde{h}(t) / R(t), \quad \delta_{d}\left(t_{m}\right)=\tilde{g}\left(t_{m}\right) / V\left(t_{m}\right), \\
& \rho_{f i}(\tau, t)=\left(\Delta_{01}^{0}(\tau, t)\right)^{2} / \Delta^{0}(t) \Delta_{11}^{0}(\tau, t) .
\end{aligned}
$$


It is obvious that the value $t^{*}$ is the value of the time lag, $\delta_{c}(t)$ and $\delta_{d}\left(t_{m}\right)$ are the signal/noise ratios with respect to the intensity in continuous and discrete channels, $\rho_{f i}(\tau, t)$ is the square of correlation coefficient between $\tilde{\mu}^{0}(t)=x_{t}-\mu^{0}(t)$ and $\tilde{\mu}^{0}(\tau, t)=x_{\tau}-\mu^{0}(\tau, t)$. Let us examine a particular case, when

$$
F(t)=-a, a>0, \tilde{h}(t)=\tilde{h}, \tilde{g}\left(t_{m}\right)=\tilde{g}, \quad R(t)=R, \quad V\left(t_{m}\right)=V,
$$

i.e., the process $x_{t}$ is Ornstein-Uhlenbeck process (Dyomin et al., 2000; Dyomin et al., 2003a, item 5), and the transmission channels are stationary with the parameters independent on time.

It is assumed: a) the point of time $t_{m}$ is the first moment of transmission in the discrete channel; b) the point of time $\tau$ is sufficiently large in order that the solution to differential equation for $\Delta^{0}(t)$ under conditions (5.69) on the interval $t \in[0, \tau]$ attain stationary value $\Delta^{0}(\tau)=\Delta^{0}=$ const.

PROPOSITION 1. $1^{0}$ ) In the general case

$$
\varepsilon_{f}\left(\tau, t_{m}\right)=\left[1+\delta_{d}\left(t_{m}\right)\left(1-\rho_{f i}\left(\tau, t_{m}-0\right)\right)\right]^{-1}
$$

$\left.2^{0}\right)$ Under conditions (5.69)

$$
\begin{aligned}
& \varepsilon_{f}\left(t^{*}\right)=\left[1+\delta_{d}\left(1-\rho_{f i}\left(t^{*}\right)\right)\right]^{-1} \\
& \rho_{f i}\left(t^{*}\right)=\left[\frac{\delta_{c}}{2\left(a+\delta_{c}\right)}+\left(1-\frac{\delta_{c}}{2\left(a+\delta_{c}\right)}\right) \exp \left\{2\left(a+\delta_{c}\right) t^{*}\right\}\right]^{-1}
\end{aligned}
$$

$\left.3^{0}\right)$ Let $\varepsilon_{f}(0)=\lim \varepsilon_{f}\left(t^{*}\right)$ as $t^{*} \downarrow 0$ and $\varepsilon_{f}(\infty)=\lim \varepsilon_{f}\left(t^{*}\right)$ as $t^{*} \uparrow \infty$. Then, $\varepsilon_{f}\left(t^{*}\right)$ is monotone decreasing function of $t^{*}$ from the value $\varepsilon_{f}(0)=1$ up to the value $\varepsilon_{f}(\infty)=\left(1+\delta_{d}\right)^{-1}$.

Proof. Formula (5.70) follows from (5.12) and (6.12)* taking into account (5.68). The solutions of $(6.10)^{*},(5.15),(5.54)$ for $\Delta^{0}(t), \Delta_{01}^{0}(\tau, t), \Delta_{11}^{0}(\tau, t)$ with the initial conditions $\Delta^{0}(\tau), \Delta_{01}^{0}(\tau, \tau)=\Delta^{0}(\tau), \Delta_{11}^{0}(\tau, \tau)=\Delta^{0}(\tau)$ under conditions (5.69) are given by

$$
\begin{aligned}
& \Delta^{0}(t)=\left[Q /\left(2 a+\delta_{c}\right)\right]+\left(\Delta^{0}(\tau)-\left[Q /\left(2 a+\delta_{c}\right)\right]\right) \exp \left\{-\left(2 a+\delta_{c}\right)(t-\tau)\right\} \\
& \Delta_{01}^{0}(\tau, t)=\Delta^{0}(\tau) \exp \left\{-\left(a+\delta_{c}\right)(t-\tau)\right\}, \\
& \Delta_{11}^{0}(\tau, t)=\Delta^{0}(\tau)\left(1-\left[\delta_{c} / 2\left(a+\delta_{c}\right)\right]\left[1-\exp \left\{-2\left(a+\delta_{c}\right)(t-\tau)\right\}\right]\right) .
\end{aligned}
$$

Since $t>\tau$, in view of the assumption b) we have $\Delta^{0}(t)=\Delta(\tau)=\Delta^{0}=Q /\left(2 a+\delta_{c}\right)$. Then, formula (5.72) arises from (5.68), (5.73) and formula (5.71) arises from (5.70) taking into account (5.72). The Property $3^{0}$ ) obviously follows from (5.71), (5.72). 
We consider the extrapolation reception under optimal manner of transmission determined by Theorems $5^{*}, 5$ and Corollary 4 , when the future value $x_{s}$ of process $x_{t}$ is predicted in the form of estimate $\mu^{0}(t, s)=M\left\{x_{s} \mid\left(z^{0}\right)_{0}^{t},\left(\eta^{0}\right)_{0}^{m}\right\}$ with mean-root-square error $\left(\Delta^{11}(t, s)\right)^{0}=M\left\{\left[x_{s}-\mu^{0}(t, s)\right]^{2}\right\}$. Then, $\varepsilon_{e}=\left(\Delta^{11}\left(t_{m}, s\right)\right)^{0} /\left(\widetilde{\Delta}^{11}\left(t_{m}, s\right)\right)^{0}$ is efficiency of discrete transmission with memory relative to discrete transmission with lag under the extrapolation reception. Let us introduce the square of corresponding correlation coefficients similar to $\rho_{f i}(\tau, t)$

$$
\rho_{f e}(t, s)=\frac{\left[\left(\Delta_{0}^{1}(t, s)\right)^{0}\right]^{2}}{\Delta^{0}(t)\left(\Delta^{11}(t, s)\right)^{0}}, \quad \rho_{i e}(\tau, t, s)=\frac{\left[\left(\Delta_{1}^{1}(\tau, t, s)\right)^{0}\right]^{2}}{\Delta_{11}^{0}(\tau, t)\left(\Delta^{11}(t, s)\right)^{0}},
$$

where $\left(\Delta_{0}^{1}(t, s)\right)^{0}=M\left\{\tilde{\mu}^{0}(t) \tilde{\mu}^{0}(t, s)\right\},\left(\Delta_{1}^{1}(\tau, t, s)\right)^{0}=M\left\{\tilde{\mu}^{0}(\tau, t) \tilde{\mu}^{0}(t, s)\right\}$, $\tilde{\mu}^{0}(t, s)=x_{s}-\mu^{0}(t, s)$.

PROPOSITION 2. On coding functional $(6.5)^{*}$ under $h(\cdot) \in \mathcal{H}_{l}$ in the case of extrapolation reception the decoding $\mu^{0}(s, t)$, the decoding error $\left(\Delta^{11}(t, s)\right)^{0}$ and $\left(\Delta_{0}^{1}(t, s)\right)^{0}$, $\left(\Delta_{1}^{1}(\tau, t, s)\right)^{0}$ on the intervals $t_{m} \leqslant t<t_{m+1}$ are defined by the equations

$$
\begin{aligned}
& \mathrm{d}_{t} \mu^{0}(t, s)=R^{-1}(t)\left[\tilde{h}(t) / \Delta^{0}(t)\right]^{1 / 2}\left(\Delta_{0}^{1}(t, s)\right)^{0} \mathrm{~d} z_{t}^{0}, \\
& \mathrm{~d}\left(\Delta^{11}(t, s)\right)^{0} / \mathrm{d} t=-\delta_{c}(t)\left(\Delta^{0}(t)\right)^{-1}\left[\left(\Delta_{0}^{1}(t, s)\right)^{0}\right]^{2}, \\
& \mathrm{~d}\left(\Delta_{0}^{1}(t, s)\right)^{0} / \mathrm{d} t=\left[F(t)+Q(t)\left(\Delta^{0}(t)\right)^{-1}-\delta_{c}(t)\right]\left(\Delta_{0}^{1}(t, s)\right)^{0}, \\
& \mathrm{~d}\left(\Delta_{1}^{1}(\tau, t, s)\right)^{0} / \mathrm{d} t=-\delta_{c}(t)\left(\Delta^{0}(t)\right)^{-1} \Delta_{01}^{0}(\tau, t)\left(\Delta_{0}^{1}(t, s)\right)^{0}
\end{aligned}
$$

with the initial conditions on coding functional $(6.6)^{*}$ under $g(\cdot) \in \mathcal{G}^{l}$

$$
\begin{aligned}
& \mu^{0}\left(t_{m}, s\right)=\mu^{0}\left(t_{m}-0, s\right) \\
& \quad+\left(\Delta_{0}^{1}\left(t_{m}-0, s\right)\right)^{0}\left[\tilde{g}\left(t_{m}\right) / \Delta^{0}\left(t_{m}-0\right)\right]^{1 / 2}\left[V\left(t_{m}\right)+\tilde{g}\left(t_{m}\right)\right]^{-1} \eta^{0}\left(t_{m}\right), \\
& \left(\Delta^{11}\left(t_{m}, s\right)\right)^{0}=\left[1+\delta_{d}\left(t_{m}\right)\right]^{-1}\left[1+\delta_{d}\left(t_{m}\right)\left(1-\rho_{f e}\left(t_{m}-0, s\right)\right)\right] \\
& \quad \times\left(\Delta^{11}\left(t_{m}-0, s\right)\right)^{0} \\
& \left(\Delta_{0}^{1}\left(t_{m}, s\right)\right)^{0}=\left[1+\delta_{d}\left(t_{m}\right)\right]^{-1}\left(\Delta_{0}^{1}\left(t_{m}-0, s\right)\right)^{0} \\
& \left(\Delta_{1}^{1}\left(\tau, t_{m}, s\right)\right)^{0}=\left[1+\delta_{d}\left(t_{m}\right)\right]^{-1} \\
& \quad \times\left[1+\delta_{d}\left(t_{m}\right)\left(1-\left[\rho_{f i}\left(\tau, t_{m}-0\right) \rho_{f e}\left(t_{m}-0, s\right) / \rho_{i e}\left(\tau, t_{m}-0, s\right)\right]^{1 / 2}\right)\right] \\
& \quad \times\left(\Delta_{1}^{1}\left(\tau, t_{m}-0, s\right)\right)^{0}
\end{aligned}
$$

and with the initial conditions on coding functional (5.6) under $g(\cdot) \in \mathcal{G}_{l}^{1}$

$$
\begin{aligned}
& \mu^{0}\left(t_{m}, s\right)=\mu^{0}\left(t_{m}-0, s\right) \\
& \quad+\left(\Delta_{1}^{1}\left(\tau, t_{m}-0, s\right)\right)^{0}\left[\tilde{g}\left(t_{m}\right) / \Delta_{11}^{0}\left(\tau, t_{m}-0\right)\right]^{1 / 2}
\end{aligned}
$$




$$
\begin{aligned}
& \quad \times\left[V\left(t_{m}\right)+\tilde{g}\left(t_{m}\right)\right]^{-1} \eta^{0}\left(t_{m}\right) \\
& \left(\Delta^{11}\left(t_{m}, s\right)\right)^{0}=\left[1+\delta_{d}\left(t_{m}\right)\right]^{-1}\left[1+\delta_{d}\left(t_{m}\right)\left(1-\rho_{i e}\left(\tau, t_{m}-0, s\right)\right)\right] \\
& \quad \times\left(\Delta^{11}\left(t_{m}-0, s\right)\right)^{0} \\
& \left(\Delta_{0}^{1}\left(t_{m}, s\right)\right)^{0}=\left[1+\delta_{d}\left(t_{m}\right)\right]^{-1} \\
& \quad \times\left[1+\delta_{d}\left(t_{m}\right)\left(1-\left[\rho_{f i}\left(\tau, t_{m}-0\right) \rho_{i e}\left(\tau, t_{m}-0, s\right) / \rho_{f e}\left(t_{m}-0, s\right)\right]^{1 / 2}\right)\right] \\
& \quad \times\left(\Delta_{0}^{1}\left(t_{m}-0, s\right)\right)^{0}, \\
& \left(\Delta_{1}^{1}\left(\tau, t_{m}, s\right)\right)^{0}=\left[1+\delta_{d}\left(t_{m}\right)\right]^{-1}\left(\Delta_{1}^{1}\left(\tau, t_{m}-0, s\right)\right)^{0} .
\end{aligned}
$$

Proof. If given $\{h(\cdot) ; g(\cdot)\} \in \mathcal{K}_{l}=\left\{\mathcal{H}_{l} ; \mathcal{G}_{l}\right\}$ of the form (6.4)* according to Corollary 2 in (Dyomin et al., 1997) $\mu(t, s)=M\left\{x_{s} \mid z_{0}^{t}, \eta_{0}^{m}\right\}, \gamma^{11}(t, s)=M\left\{\tilde{\mu}(t, s)^{2} \mid z_{0}^{t}, \eta_{0}^{m}\right\}$, $\gamma_{0}^{1}(t, s)=M\left\{\tilde{\mu}(t) \tilde{\mu}(t, s) \mid z_{0}^{t}, \eta_{0}^{m}\right\}, \gamma_{1}^{1}(\tau, t, s)=M\left\{\tilde{\mu}(\tau, t) \tilde{\mu}(t, s) \mid z_{0}^{t}, \eta_{0}^{m}\right\}$, where $\tilde{\mu}(t, s)=x_{s}-\mu(t, s), \tilde{\mu}(t)=x_{t}-\mu(t), \tilde{\mu}(\tau, t)=x_{\tau}-\mu(\tau, t), \mu(t)=M\left\{x_{t} \mid z_{0}^{t}, \eta_{0}^{m}\right\}$, $\mu(\tau, t)=M\left\{x_{\tau} \mid z_{0}^{t}, \eta_{0}^{m}\right\}$ on the intervals $t_{m} \leqslant t<t_{m+1}$ are defined by the equations

$$
\begin{aligned}
& \mathrm{d}_{t} \mu(t, s)=R^{-1}(t)\left[H_{0}(t, z) \gamma_{0}^{1}(t, s)+H_{1}(t, z) \gamma_{1}^{1}(\tau, t, s)\right] \mathrm{d} \tilde{z}_{t}, \\
& \mathrm{~d} \gamma^{11}(t, s) / \mathrm{d} t=-R^{-1}(t)\left[H_{0}(t, z) \gamma_{0}^{1}(t, s)+H_{1}(t, z) \gamma_{1}^{1}(\tau, t, s)\right]^{2}, \\
& \mathrm{~d} \gamma_{0}^{1}(t, s) / \mathrm{d} t=\left[F(t)+Q(t) \gamma^{-1}(t)\right] \gamma_{0}^{1}(t, s) \\
& \quad-R^{-1}(t)\left[H_{0}(t, z) \gamma(t)+H_{1}(t, z) \gamma_{01}(\tau, t)\right] \\
& \quad \times\left[H_{0}(t, z) \gamma_{0}^{1}(t, s)+H_{1}(t, z) \gamma_{1}^{1}(\tau, t, s)\right], \\
& \mathrm{d} \gamma_{1}^{1}(\tau, t, s) / \mathrm{d} t=-R^{-1}(t)\left[H_{0}(t, z) \gamma_{01}(\tau, t)+H_{1}(t, z) \gamma_{11}(\tau, t)\right] \\
& \quad \times\left[H_{0}(t, z) \gamma_{0}^{1}(t, s)+H_{1}(t, z) \gamma_{1}^{1}(\tau, t, s)\right], \\
& \mathrm{d} \tilde{z}_{t}=\mathrm{d} z_{t}-\left[h(t, z)+H_{0}(t, z) \mu(t)+H_{1}(t, z) \mu(\tau, t)\right] \mathrm{d} t,
\end{aligned}
$$

with initial conditions

$$
\begin{aligned}
& \mu\left(t_{m}, s\right)=\mu\left(t_{m}-0, s\right) \\
& \quad+\left[G_{0}\left(t_{m}, z\right) \gamma_{0}^{1}\left(t_{m}-0, s\right)+G_{1}\left(t_{m}, z\right) \gamma_{1}^{1}\left(\tau, t_{m}-0, s\right)\right] W^{-1}\left(t_{m}, z\right) \tilde{\eta}\left(t_{m}\right), \\
& \gamma^{11}\left(t_{m}, s\right)=\gamma^{11}\left(t_{m}-0, s\right) \\
& \quad-\left[G_{0}\left(t_{m}, z\right) \gamma_{0}^{1}\left(t_{m}-0, s\right)+G_{1}\left(t_{m}, z\right) \gamma_{1}^{1}\left(\tau, t_{m}-0, s\right)\right]^{2} W^{-1}\left(t_{m}, z\right), \\
& \gamma_{0}^{1}\left(t_{m}, s\right)=\gamma_{0}^{1}\left(t_{m}-0, s\right)-\left[G_{0}\left(t_{m}, z\right) \gamma\left(t_{m}-0\right)+G_{1}\left(t_{m}, z\right) \gamma_{01}\left(\tau, t_{m}-0\right)\right] \\
& \quad \times\left[G_{0}\left(t_{m}, z\right) \gamma_{0}^{1}\left(t_{m}-0, s\right)+G_{1}\left(t_{m}, z\right) \gamma_{1}^{1}\left(\tau, t_{m}-0, s\right)\right] W^{-1}\left(t_{m}, z\right), \\
& \gamma_{1}^{1}\left(\tau, t_{m}, s\right)=\gamma_{1}^{1}\left(\tau, t_{m}-0, s\right) \\
& \quad-\left[G_{0}\left(t_{m}, z\right) \gamma_{01}\left(\tau, t_{m}-0\right)+G_{1}\left(t_{m}, z\right) \gamma_{11}\left(\tau, t_{m}-0\right)\right] \\
& \quad \times\left[G_{0}\left(t_{m}, z\right) \gamma_{0}^{1}\left(t_{m}-0, s\right)+G_{1}\left(t_{m}, z\right) \gamma_{1}^{1}\left(\tau, t_{m}-0, s\right)\right] W^{-1}\left(t_{m}, z\right), \\
& \tilde{\eta}\left(t_{m}\right)=\eta\left(t_{m}\right)-\left[g\left(t_{m}, z\right)+G_{0}\left(t_{m}, z\right) \mu\left(t_{m}-0\right)+G_{1}\left(t_{m}, z\right) \mu\left(\tau, t_{m}-0\right)\right], \\
& W\left(t_{m}, z\right)=V\left(t_{m}\right)+G_{0}^{2}\left(t_{m}, z\right) \gamma\left(t_{m}-0\right)+G_{1}^{2}\left(t_{m}, z\right) \gamma_{11}\left(\tau, t_{m}-0\right) \\
& \quad+2 G_{0}\left(t_{m}, z\right) G_{1}\left(t_{m}, z\right) \gamma_{01}\left(\tau, t_{m}-0\right) .
\end{aligned}
$$


The use of $h^{0}(\cdot)$ in the form $(6.5)^{*}$ into (5.87) brings us to (5.75)-(5.78). Using $g^{0}(\cdot)$ in the form $(6.6)^{*}$, and then, in the form (5.6) into (5.88), we obtain (5.79)-(5.82) and (5.83)-(5.86).

REMARK 10. Formulae $(6.12)^{*}$ and (5.12) can be represented in the form

$$
\begin{aligned}
& \Delta^{0}\left(t_{m}\right)=\left[1+\delta_{d}\left(t_{m}\right)\right]^{-1} \Delta^{0}\left(t_{m}-0\right) \\
& \Delta^{0}\left(t_{m}\right)=\left[1+\delta_{d}\left(t_{m}\right)\right]^{-1}\left[1+\delta_{d}\left(t_{m}\right)\left(1-\rho_{f i}\left(\tau, t_{m}-0\right)\right)\right] \Delta^{0}\left(t_{m}-0\right) .
\end{aligned}
$$

For $s=t$, we have $\mu^{0}(t, s)=\mu^{0}(t),\left(\Delta_{0}^{1}(t, s)\right)^{0}=\left(\Delta^{11}(t, s)\right)^{0}=\Delta^{0}(t)$, $\left(\Delta_{1}^{1}(\tau, t, s)\right)^{0}=\Delta_{01}^{0}(\tau, t), \rho_{f e}(t, s)=1, \rho_{i e}(\tau, t, s)=\rho_{f i}(\tau, t)$. Then, formulae (5.79), (5.80), (5.83), (5.84) have the form $(6.11)^{*},(5.89),(5.11),(5.90)$, respectively. This result is explained by the fact that for $s=t$ extrapolation reception is changing over to filtering reception.

Proposition 3. $1^{0}$ ) In the general case

$$
\begin{aligned}
\varepsilon_{e}\left(\tau, t_{m}, s\right)= & {\left[1+\delta_{d}\left(t_{m}\right)\left(1-\rho_{f e}\left(t_{m}-0, s\right)\right)\right] } \\
& \times\left[1+\delta_{d}\left(t_{m}\right)\left(1-\rho_{i e}\left(\tau, t_{m}-0, s\right)\right)\right]^{-1} .
\end{aligned}
$$

$\left.2^{0}\right)$ Suppose that $T=s-t$ is value of time interval of extrapolation. Then, under conditions (5.69)

$$
\begin{aligned}
& \varepsilon_{e}\left(t^{*}, T\right)=\left[1+\delta_{d}\left(1-\rho_{f e}(T)\right)\right]\left[1+\delta_{d}\left(1-\rho_{i e}\left(t^{*}, T\right)\right)\right]^{-1}, \\
& \rho_{i e}\left(t^{*}, T\right)=\rho_{f i}\left(t^{*}\right) \rho_{f e}(T) \\
& \rho_{f e}(T)=\left[1+\left(1+\left(\delta_{c} / 2 a\right)\right)(\exp \{2 a T\}-1)\right]^{-1}
\end{aligned}
$$

where $\rho_{f i}\left(t^{*}\right)$ determined by Proposition 1 .

$\left.3^{0}\right)$ Let $\varepsilon_{e}(0, T)=\lim \varepsilon_{e}\left(t^{*}, T\right)$ as $t^{*} \downarrow 0$ and $\varepsilon_{e}(\infty, T)=\lim \varepsilon_{e}\left(t^{*}, T\right)$ as $t^{*} \uparrow \infty$. Then, $\varepsilon_{e}\left(t^{*}, T\right)$ is monotone decreasing function of $t^{*}$ from the value $\varepsilon_{e}(0, T)=1$ up to the value $\varepsilon_{e}(\infty, T)=\left(1+\delta_{d}\right)^{-1}\left[1+\delta_{d}\left(1-\rho_{f e}(T)\right)\right]$.

$\left.4^{0}\right)$ Let $\varepsilon_{e}\left(t^{*}, 0\right)=\lim \varepsilon_{e}\left(t^{*}, T\right)$ as $T \downarrow 0$ and $\varepsilon_{e}\left(t^{*}, \infty\right)=\lim \varepsilon_{e}\left(t^{*}, T\right)$ as $T \uparrow \infty$. Then, $\varepsilon_{e}\left(t^{*}, T\right)$ is monotone increasing function of $T$ from the value $\varepsilon_{e}\left(t^{*}, 0\right)=\varepsilon_{f}\left(t^{*}\right)$ of the form (5.71) up to the value $\varepsilon_{e}\left(t^{*}, \infty\right)=1$.

Proof. Formula (5.91) follows from (5.74), (5.80), (5.84). The solutions of (5.76)-(5.78) with the initial conditions $\left(\Delta^{11}(s, s)\right)^{0}=\Delta^{0}(s),\left(\Delta_{0}^{1}(s, s)\right)^{0}=\Delta^{0}(s),\left(\Delta_{1}^{1}(\tau, s, s)\right)^{0}=$ $\Delta_{01}^{0}(\tau, s)$ under conditions $(5.69)$ are given by

$$
\begin{aligned}
& \left(\Delta^{11}(t, s)\right)^{0}=\Delta^{0}(t) \exp \{-2 a(s-t)\}+(Q / 2 a)[1-\exp \{-2 a(s-t)\}] \\
& \left(\Delta_{0}^{1}(t, s)\right)^{0}=\Delta^{0}(t) \exp \{-a(s-t)\} \\
& \left(\Delta_{1}^{1}(\tau, t, s)\right)^{0}=\Delta^{0}(\tau) \exp \left\{-\left(a+\delta_{c}\right)(t-\tau)\right\} \exp \{-a(s-t)\} .
\end{aligned}
$$


Then, analogously to (5.71), (5.72) formulae (5.92)-(5.94) arise from (5.74), (5.91), (5.95). The properties $\left.\left.3^{0}\right), 4^{0}\right)$ obviously follow from (5.71), (5.72), (5.92)-(5.94).

As measures of information efficiency for the cases of filtering and extrapolation receptions the values $\Delta_{f}=\Delta I_{t_{m}}\left[x_{t_{m}} ;\left(z^{0}\right)_{0}^{t_{m}}, \eta^{0}\left(t_{m}\right)\right]-\Delta \widetilde{I}_{t_{m}}\left[x_{t_{m}} ;\left(z^{0}\right)_{0}^{t_{m}}, \eta^{0}\left(t_{m}\right)\right]$ and $\Delta_{e}=\Delta I_{s}^{t_{m}}\left[x_{s} ;\left(z^{0}\right)_{0}^{t_{m}}, \eta^{0}\left(t_{m}\right)\right]-\Delta \widetilde{I}_{s}^{t_{m}}\left[x_{s} ;\left(z^{0}\right)_{0}^{t_{m}}, \eta^{0}\left(t_{m}\right)\right]$ are taken.

Proposition 4. $1^{0}$ ) In the general case

$$
\begin{aligned}
& \Delta_{f}\left(\tau, t_{m}\right)=(1 / 2) \ln \left\{\left[1+\delta_{d}\left(t_{m}\right)\left(1-\rho_{f i}\left(\tau, t_{m}-0\right)\right)\right]\right\} \\
& \Delta_{e}\left(\tau, t_{m}, s\right)=(1 / 2) \ln \left\{\left[1+\delta_{d}\left(t_{m}\right)\left(1-\rho_{i e}\left(\tau, t_{m}-0, s\right)\right)\right]\right. \\
& \left.\quad \times\left[1+\delta_{d}\left(t_{m}\right)\left(1-\rho_{f e}\left(t_{m}-0, s\right)\right)\right]^{-1}\right\} .
\end{aligned}
$$

$\left.2^{0}\right)$ Under conditions (5.69)

$$
\begin{aligned}
& \Delta_{f}\left(t^{*}\right)=(1 / 2) \ln \left\{\left[1+\delta_{d}\left(1-\rho_{f i}\left(t^{*}\right)\right)\right]\right\} \\
& \Delta_{e}\left(t^{*}, T\right)=(1 / 2) \ln \left\{\left[1+\delta_{d}\left(1-\rho_{i e}\left(t^{*}, T\right)\right)\right]\left[1+\delta_{d}\left(1-\rho_{f e}(T)\right)\right]^{-1}\right\}
\end{aligned}
$$

where $\rho_{f i}\left(t^{*}\right), \rho_{f e}(T), \rho_{i e}\left(t^{*}, T\right)$ determined by Propositions 1 and 3 .

$\left.3^{0}\right)$ Let: $\Delta_{f}(0)=\lim \Delta_{f}\left(t^{*}\right)$ and $\Delta_{e}(0, T)=\lim \Delta_{e}\left(t^{*}, T\right)$ as $t^{*} \downarrow 0 ; \Delta_{f}(\infty)=$ $\lim \Delta_{f}\left(t^{*}\right)$ and $\Delta_{e}(\infty, T)=\lim \Delta_{e}\left(t^{*}, T\right)$ as $t^{*} \uparrow \infty$. Then, $\Delta_{f}\left(t^{*}\right)$ and $\Delta_{e}\left(t^{*}, T\right)$ are monotone increasing functions of $t^{*}$ from the value $\Delta_{f}(0)=0$ and $\Delta_{e}(0, T)=0$ up to the values $\Delta_{f}(\infty)=(1 / 2) \ln \left\{\left(1+\delta_{d}\right)\right\}$ and $\Delta_{e}(\infty, T)=(1 / 2) \ln \left\{\left(1+\delta_{d}\right)\left[1+\delta_{d}(1-\right.\right.$ $\left.\left.\left.\rho_{f e}(T)\right)\right]^{-1}\right\}$.

$\left.4^{0}\right)$ Let $\Delta_{e}\left(t^{*}, 0\right)=\lim \Delta_{e}\left(t^{*}, T\right)$ as $T \downarrow 0$ and $\Delta_{e}\left(t^{*}, \infty\right)=\lim \Delta_{e}\left(t^{*}, T\right)$ as $T \uparrow$ $\infty$. Then, $\Delta_{e}\left(t^{*}, T\right)$ is monotone decreasing function of $T$ from the value $\Delta_{e}\left(t^{*}, 0\right)=$ $\Delta_{f}\left(t^{*}\right)$ up to the value $\Delta_{e}\left(t^{*}, \infty\right)=0$.

Proof. Formulae (5.96) arise from $(4.34)^{*},(4.36)^{*},(5.80),(5.84),(5.89),(5.90)$. Formulae (5.97) and Properties $\left.\left.3^{0}\right), 4^{0}\right)$ arise from (5.96) taking into account (5.72), (5.93), (5.94). In the case of $s=t$ and $T=0$, when extrapolation reception is changing over to filtering reception, the formulae for $\Delta_{e}\left(\tau, t_{m}, s\right)$ and $\Delta_{e}\left(t^{*}, T\right)$ pass into formulae for $\Delta_{f}\left(\tau, t_{m}\right)$ and $\Delta_{f}\left(t^{*}\right)$ (see Remark 10).

Let us comment on the results obtained for cases, when the conditions (5.69) and assumption b) take place.

1. The efficiency of discrete channels with and without memory (see Remark 9) relatively the channel with lag is defined by the ratios signal/noise by the intensity in the continuous channel $\delta_{c}$, in the discrete channel $\delta_{d}$ and by the square of correlation coefficients $\rho_{f i}\left(t^{*}\right), \rho_{f e}(T), \rho_{i e}\left(t^{*}, T\right)$ that depend on the value of time lag $t^{*}$ within the signal transmission $x_{t}$ in the discrete channel with lag and the value of the time extrapolation interval $T$ by the extrapolation reception. 
2. Equalities $\varepsilon_{f}(0)=\varepsilon_{e}(0, T)=1$ and $\Delta_{f}(0)=\Delta_{e}(0, T)=0$ are obvious, since the channel with lag is changing over to channel without lag by $t^{*}=0$. Monotone decrease $\varepsilon_{f}\left(t^{*}\right), \varepsilon_{e}\left(t^{*}, T\right)$ and monotone increase $\Delta_{f}\left(t^{*}\right), \Delta_{e}\left(t^{*}, T\right)$ of $t^{*}$ are explained by the fact that while $t^{*}$ is increasing, the statistic dependencies between $x_{\tau}$ and $x_{t_{m}}, x_{\tau}$ and $x_{s}$ are decreasing. This effect lead to decrease of information amount about the current $x_{t_{m}}$ and future $x_{s}$ values of the process $x_{t}$, which is contained in the past value $x_{\tau}$ transmitted in the channel with lag.

3. Equalities $\varepsilon_{e}\left(t^{*}, 0\right)=\varepsilon_{f}\left(t^{*}\right)$ and $\Delta_{e}\left(t^{*}, 0\right)=\Delta_{f}\left(t^{*}\right)$ are explained by the fact that if $T=0$ then an extrapolation reception is changing over to filtering reception. Monotone increase $\varepsilon_{e}\left(t^{*}, T\right)$ up to unity and monotone decrease $\Delta_{e}\left(t^{*}, T\right)$ up to zero by the increasing of $T$ are explained by decreasing statistic dependencies up to zero between $x_{s}$ and $x_{t_{m}}, x_{s}$ and $x_{\tau}$, that results in equivalence of discrete channels with memory and lag.

4. The influence of a continuous transmission channel into discrete channels is being implemented by a parameter $\delta_{c}$. Absence of continuous transmission corresponds to the case $\delta_{c}=0$, and ideal (noiseless) continuous transmission corresponds to the case $\delta_{c}=\infty$. Let $\lim \varepsilon_{f}\left(t^{*}\right)=\varepsilon_{f}^{0}\left(t^{*}\right), \lim \varepsilon_{e}\left(t^{*}, T\right)=\varepsilon_{e}^{0}\left(t^{*}, T\right), \lim \Delta_{f}\left(t^{*}\right)=\Delta_{f}^{0}\left(t^{*}\right)$, $\lim \Delta_{e}\left(t^{*}, T\right)=\Delta_{e}^{0}\left(t^{*}, T\right)$ as $\delta_{c} \downarrow 0$, and $\lim \varepsilon_{f}\left(t^{*}\right)=\varepsilon_{f}^{\infty}\left(t^{*}\right), \lim \varepsilon_{e}\left(t^{*}, T\right)=$ $\varepsilon_{e}^{\infty}\left(t^{*}, T\right), \lim \Delta_{f}\left(t^{*}\right)=\Delta_{f}^{\infty}\left(t^{*}\right), \lim \Delta_{e}\left(t^{*}, T\right)=\Delta_{e}^{\infty}\left(t^{*}, T\right)$ as $\delta_{c} \uparrow \infty$. From (5.72), (5.93), (5.94) follows that by $\delta_{c}=0$

$$
\begin{aligned}
& \rho_{f i}\left(t^{*}\right)=\rho_{f i}^{0}\left(t^{*}\right)=\exp \left\{-2 a t^{*}\right\}, \quad \rho_{f e}(T)=\rho_{f e}^{0}(T)=\exp \{-2 a T\}, \\
& \rho_{i e}\left(t^{*}, T\right)=\rho_{i e}^{0}\left(t^{*}, T\right)=\exp \left\{-2 a\left(t^{*}+T\right)\right\},
\end{aligned}
$$

i.e., with the absence of continuous observations $\rho_{f i}, \rho_{f e}, \rho_{i e}$ are changing over to square of correlation coefficients between the appropriate values of the process $x_{t}$. Thus if $\delta_{c}=$ 0 then Propositions 1, 3, 4 take place, where instead of (5.72),(5.93), (5.94), formulae (5.98), are used. Analogously, $\rho_{f i}=\rho_{f e}=\rho_{i e}=0$ by $\delta_{c}=\infty$. Then, from (5.71), (5.92), (5.97) follows that $\varepsilon_{f}^{\infty}\left(t^{*}\right)=\left(1+\delta_{d}\right)^{-1}<\varepsilon_{f}\left(t^{*}\right), \Delta_{f}^{\infty}\left(t^{*}\right)=(1 / 2) \ln \{(1+$ $\left.\left.\delta_{d}\right)\right\}>\Delta_{f}\left(t^{*}\right), \varepsilon_{e}^{\infty}\left(t^{*}, T\right)=1, \Delta_{e}^{\infty}\left(t^{*}, T\right)=0$, i.e., the ideal continuous channel with a noiseless feedback increases discrete channel efficiency with memory relatively the channel with lag within filtering reception and makes them equivalent within the extrapolation transmission.

5. Let $\varepsilon_{f_{0}}\left(t^{*}\right)=\lim \varepsilon_{f}\left(t^{*}\right), \varepsilon_{e_{0}}\left(t^{*}, T\right)=\lim \varepsilon_{e}\left(t^{*}, T\right), \Delta_{f_{0}}\left(t^{*}\right)=\lim \Delta_{f}\left(t^{*}\right)$, $\Delta_{e_{0}}\left(t^{*}, T\right)=\lim \Delta_{e}\left(t^{*}, T\right)$ as $\delta_{d} \downarrow 0$, and $\varepsilon_{f_{\infty}}\left(t^{*}\right)=\lim \varepsilon_{f}\left(t^{*}\right), \varepsilon_{e_{\infty}}\left(t^{*}, T\right)=$ $\lim \varepsilon_{e}\left(t^{*}, T\right), \Delta_{f_{\infty}}\left(t^{*}\right)=\lim \Delta_{f}\left(t^{*}\right), \Delta_{e_{\infty}}\left(t^{*}, T\right)=\lim \Delta_{e}\left(t^{*}, T\right)$ as $\delta_{d} \uparrow \infty$. From Propositions 1, 3, 4 it follows that $\varepsilon_{f_{0}}\left(t^{*}\right)=1, \Delta_{f_{0}}\left(t^{*}\right)=0, \varepsilon_{e_{0}}\left(t^{*}, T\right)=1$, $\Delta_{e_{0}}\left(t^{*}, T\right)=0$, i.e., by unlimited increasing noise intensity relatively the signal intensity in discrete channels they become equivalent, since both of them no contain information about the process $x_{t}$. In a similar manner $\varepsilon_{f_{\infty}}\left(t^{*}\right)=0, \Delta_{f_{\infty}}\left(t^{*}\right)=\infty$, $\varepsilon_{e_{\infty}}\left(t^{*}, T\right)=\left[1-\rho_{f e}(T)\right]\left[1-\rho_{i e}\left(t^{*}, T\right)\right]^{-1}<\varepsilon_{e}\left(t^{*}, T\right), \Delta_{e_{\infty}}\left(t^{*}, T\right)=(1 / 2) \ln \{[1-$ $\left.\left.\rho_{i e}\left(t^{*}, T\right)\right]\left[1-\rho_{f e}(T)\right]^{-1}\right\}>\Delta_{e}\left(t^{*}, T\right)$, that is ideal (noiseless) discrete channel increases efficiency of the discrete channel with memory relatively to the channel with lag, moreover, in the case of filtering reception - unrestrictedly. 
6. Suppose $\varepsilon_{f e}\left(t_{m}\right)=\Delta^{0}\left(t_{m}\right) /\left(\Delta^{11}\left(t_{m}, s\right)\right)^{0}, \Delta I_{f e}\left(t_{m}\right)=I_{t_{m}}[\cdot]-I_{s}^{t_{m}}[\cdot]$ characterize filtering reception efficiency relatively to extrapolation reception in discrete channels. Then,

$$
\begin{aligned}
& \varepsilon_{f e}\left(t_{m}\right)=\left[1+\delta_{d}\left(1-\rho_{f e}(T)\right)\right]^{-1} \varepsilon_{f e}(T) \\
& \Delta I_{f e}\left(t_{m}\right)=\frac{1}{2} \ln \left\{\left[1+\delta_{d}\left(1-\rho_{f e}(T)\right)\right]\right\}+\Delta I_{f e}(T)
\end{aligned}
$$

in the case of discrete channel with memory and

$$
\begin{gathered}
\varepsilon_{f e}\left(t_{m}\right)=\left[1+\delta_{d}\left(1-\rho_{f i}\left(t^{*}\right)\right)\right]\left[1+\delta_{d}\left(1-\rho_{i e}\left(t^{*}, T\right)\right)\right]^{-1} \varepsilon_{f e}(T) \\
\Delta I_{f e}\left(t_{m}\right)=\frac{1}{2} \ln \left\{\left[1+\delta_{d}\left(1-\rho_{i e}\left(t^{*}, T\right)\right)\right]\left[1+\delta_{d}\left(1-\rho_{f i}\left(t^{*}\right)\right)\right]^{-1}\right\} \\
+\Delta I_{f e}(T)
\end{gathered}
$$

in the case of discrete channel with lag, where $\varepsilon_{f e}(T)=\left[\left(1+\left(\delta_{c} / 2 a\right)\right)-\right.$ $\left.\left(\delta_{c} / 2 a\right) \exp \{-2 a T\}\right]^{-1}$ and $\Delta I_{f e}(T)=(1 / 2) \ln \left\{\varepsilon_{f e}^{-1}(T)\right\}$ are the characteristics of filtering reception efficiency relatively extrapolation reception in a continuous channel. Formulas (5.99), (5.100) follow from the previous results. It is obvious that $\varepsilon_{f e}(T)<1$, $\Delta I_{f e}(T)>0, \varepsilon_{f e}\left(t_{m}\right)<\varepsilon_{f e}(T)<1, \Delta I_{f e}\left(t_{m}\right)>\Delta I_{f e}(T)>0$, that is a filtering reception is more efficient then extrapolation reception one both in continuous and discrete transmission channels.

\section{Conclusion}

The results obtained and (Dyomin et al., 2003a) allow us to point out some problems which are open for future investigations.

1. The finding of Shannon information amount in the joint filtering, extrapolation and interpolation problem of stochastic processes by continuous-discrete time memory observations and the investigation of this information amount structure. The consideration of these problems for cases of moving memory and moving extrapolation on basis of (Dyomin et al., 2000).

2. The investigation of optimal transmission problems of stochastic processes by memory continuous-discrete channels without the silent feedback and the comparison of results to be obtained with the given.

3. The investigation of optimal transmission problems of stochastic processes by memory continuous-discrete channels both with and without the feedback when general energy of message is distributed among the signals which transmit current and past values of the process. 


\section{References}

Abakumova, O.L., N.S. Dyomin and T.V. Sushko (1995a). Filtering of stochastic processes for continuous and discrete observations with memory I. Main equation of nonlinear filtering. Avtom. Telemekh., 9, 49-59 (in Russian).

Abakumova, O.L., N.S. Dyomin and T.V. Sushko (1995b). Filtering of stochastic processes for continuous and discrete observations with memory II. Synthesis of filters. Automat. and Remote Control, 56(10), 1383-1393.

Dyomin, N.S., and V.I. Korotkevich (1983). Amount of information in problems of filtering the components of Markov processes. Autom. Remote Control, 44(7), 899-907.

Dyomin, N.S., and V.I. Korotkevich (1987). Equations for Shannon information on transmission of Markov diffusion signals through channels with memory. Problems of Inform. Trans., 23(1), 11-21.

Dyomin, N.S., S.V. Rozhkova and O.V. Rozhkova (2000). Generalized moving extrapolation of stochastic processes based on the totality of continuous and discrete observations with memory. J. Comput. Syst. Sci. Intern., 39(4), 523-535.

Dyomin, N.S., I.E. Safronova and S.V. Rozhkova (2003a). Information amount determination for joint problem of filtering and generalized extrapolation of stochastic processes with respect to the set of continuous and discrete memory observations. Informatica, 14(3), 295-322.

Dyomin, N.S., I.E. Safronova and S.V. Rozhkova (2003b). The information analysis in joint problem of continuous-discrete filtering and generalized extrapolation. In 13th IFAC Symp. on Syst. Ident., Preprints. pp. $1048-1053$.

Dyomin, N.S., T.V. Sushko and A.V. Yakovleva (1997). Generalized inverse extrapolation of stochastic processes by an aggregate of continuous-discrete observations with memory. J. Comput. Syst. Sci. Intern., 36(4), $543-554$

Gallager, R.G. (1968). Information Theory and Reliable Communication. Wiley, New York.

Gantmakher, F.R. (1988). Theory of Matrices. Nauka, Moscow (in Russian).

Liptser, R.Sh. (1974). Optimal coding and decoding for transmission of Gaussian Markov signal over noiseless feedback channel. Probl. Peredachi Inf., 10(4), 3-15 (in Russian).

Liptser, R.Sh., and A.N. Shiryayev (1977, 1978). Statistics of Random Processes. Springer Verlag, New York.

Ocone, D., and E. Pardoux (1989). A generelized Ito-Ventzel formula. Ann. Inst. Henri Poincare, 25(1), 39-71.

Shannon, C.E., and W. Weaver (1949). The Mathematical Theory of Communication. Univ. Illinois Press, Urbana, Illinois. 
N. Dyomin graduated from Department of Radio and Physics, Tomsk State University, Russia, in 1967. He received the PhD degree in technical cybernetics and information theory from Tomsk State University in 1977 and the DrSci degree in application of mathematical methods in science investigations from the same university in 1990. He is an full professor in Department of Applied Mathematics and Cybernetics of Tomsk State University. Present research interests: filtering, interpolation, extrapolation and recognition of stochastic processes; transmission of stochastic signals along continuous-discrete observation memory channels; detection of anomalous noises; mathematical theory of financial analysis and security trading.

S. Rozhkova graduated from Department of Applied Mathematics and Cybernetics of Tomsk State University, Russia, in 1993. She received the PhD degree in application of mathematical methods in science investigations from Tomsk State University in 1996. She is an associated professor in Department of Natural Sciences and Mathematics of Tomsk Polytechnic University. Present research interests: filtering, interpolation, extrapolation and recognition of stochastic processes; transmission of stochastic signals along continuous-discrete observation memory channels; detection of anomalous noises.

I. Safronova graduated from Department of Applied Mathematics and Cybernetics of Tomsk State University, Russia, in 2000. She is a post-graduate in Department of Applied Mathematics and Cybernetics of Tomsk State University. Present research interests: filtering, interpolation, extrapolation of stochastic processes; transmission of stochastic signals along continuous-discrete observation memory channels. 


\section{Apie Šenono informacijos kiekio atsitiktiniu procesu bendrame filtracijos ir interpoliacijos uždavinyje struktūrą tolydžiais-diskrečiais laiko momentais}

Nikolas DYOMIN, Irina SAFRONOVA, Svetlana ROZHKOVA

Naudojantis darbo (Dyomin et al., 2003a) rezultatais, šiame straipsnyje yra nagrinejjama Šenono informacijos kiekio atsitiktiniu procesų bendrame filtracijos ir interpoliacijos uždavinyje struktūra. Tiriamas atvejis, kai viena dalis komponenčiu yra stebima diskrečiais laiko momentais, o kita dalis - tolydžiu laiku. Specialiai procesų klasei yra sprendžiamas optimalaus perdavimo kanalais su vèlavimu uždavinys, o taip pat tiriamas prièmimo filtracijos ir ekstrapoliacijos efektyvumas, kai perduodama kanalais su atmintimi arba su vèlavimu. 